APS

physics

This is the accepted manuscript made available via CHORUS. The article has been published as:

\title{
Visualizing spacetime curvature via frame-drag vortexes and tidal tendexes: General theory and weak-gravity applications
}

David A. Nichols, Robert Owen, Fan Zhang, Aaron Zimmerman, Jeandrew Brink, Yanbei Chen, Jeffrey D. Kaplan, Geoffrey Lovelace, Keith D. Matthews, Mark A. Scheel, and Kip S. Thorne

Phys. Rev. D 84, 124014 - Published 6 December 2011 DOI: 10.1103/PhysRevD.84.124014 


\title{
Visualizing Spacetime Curvature via Frame-Drag Vortexes and Tidal Tendexes I. General Theory and Weak-Gravity Applications
}

\author{
David A. Nichols, ${ }^{1}$ Robert Owen, ${ }^{2}$ Fan Zhang, ${ }^{1}$ Aaron Zimmerman, ${ }^{1}$ Jeandrew Brink,${ }^{3}$ Yanbei Chen, ${ }^{1}$ \\ Jeffrey D. Kaplan, ${ }^{1}$ Geoffrey Lovelace, ${ }^{2}$ Keith D. Matthews, ${ }^{1}$ Mark A. Scheel, ${ }^{1}$ and Kip S. Thorne ${ }^{1,4}$ \\ ${ }^{1}$ Theoretical Astrophysics 350-17, California Institute of Technology, Pasadena, California 91125, USA \\ ${ }^{2}$ Center for Radiophysics and Space Research, Cornell University, Ithaca, New York 14853, USA \\ ${ }^{3}$ National Institute of Theoretical Physics, Private Bag X1 Matieland, Stellenbosch 7602, South Africa \\ 4 Stellenbosch Institute for Advanced Study (STIAS), Wallenberg Research \\ Centre at Stellenbosch University, Marais Street, Stellenbosch 7600, South Africa
}

When one splits spacetime into space plus time, the Weyl curvature tensor (vacuum Riemann tensor) gets split into two spatial, symmetric, and trace-free (STF) tensors: (i) the Weyl tensor's so-called "electric" part or tidal field $\mathcal{E}_{j k}$, which raises tides on the Earth's oceans and drives geodesic deviation (the relative acceleration of two freely falling test particles separated by a spatial vector $\xi^{k}$ is $\Delta a_{j}=-\mathcal{E}_{j k} \xi^{k}$ ); and (ii) the Weyl tensor's so-called "magnetic" part or (as we call it) frame-drag field $\mathcal{B}_{j k}$, which drives differential frame dragging (the precessional angular velocity of a gyroscope at the tip of $\xi^{k}$, as measured using a local inertial frame at the tail of $\xi^{k}$, is $\Delta \Omega_{j}=\mathcal{B}_{j k} \xi^{k}$.)

Being STF, $\mathcal{E}_{j k}$ and $\mathcal{B}_{j k}$ each have three orthogonal eigenvector fields which can be depicted by their integral curves. We call the integral curves of $\mathcal{E}_{j k}$ 's eigenvectors tidal tendex lines or simply tendex lines, we call each tendex line's eigenvalue its tendicity, and we give the name tendex to a collection of tendex lines with large tendicity. The analogous quantities for $\mathcal{B}_{j k}$ are frame-drag vortex lines or simply vortex lines, their vorticities, and vortexes.

These concepts are powerful tools for visualizing spacetime curvature. We build up physical intuition into them by applying them to a variety of weak-gravity phenomena: a spinning, gravitating point particle, two such particles side by side, a plane gravitational wave, a point particle with a dynamical current-quadrupole moment or dynamical mass-quadrupole moment, and a slow-motion binary system made of nonspinning point particles. We show that a rotating current quadrupole has four rotating vortexes that sweep outward and backward like water streams from a rotating sprinkler. As they sweep, the vortexes acquire accompanying tendexes and thereby become outgoing current-quadrupole gravitational waves. We show similarly that a rotating mass quadrupole has four rotating, outward and backward sweeping tendexes that acquire accompanying vortexes as they sweep, and become outgoing mass-quadrupole gravitational waves. We show, further, that an oscillating current quadrupole ejects sequences of vortex loops that acquire accompanying tendex loops as they travel, and become current-quadrupole gravitational waves; and similarly for an oscillating mass quadrupole. And we show how a binary's tendex lines transition, as one moves radially, from those of two static point particles in the deep near zone, to those of a single spherical body in the outer part of the near zone and inner part of the wave zone (where the binary's mass monopole moment dominates), to those of a rotating quadrupole in the far wave zone (where the quadrupolar gravitational waves dominate).

In paper II we will use these vortex and tendex concepts to gain insight into the quasinormal modes of black holes, and in subsequent papers, by combining these concepts with numerical simulations, we will explore the nonlinear dynamics of curved spacetime around colliding black holes. We have published a brief overview of these applications in Physical Review Letters [1]. We expect these vortex and tendex concepts to become powerful tools for general relativity research in a variety of topics.

PACS numbers: 04.25.dg, 04.25.Nx, 04.30.-w

\section{MOTIVATION AND OVERVIEW}

In the 1950s John Archibald Wheeler coined the phrase geometrodynamics to epitomize his intuition that curved spacetime must have a rich range of nonlinear dynamical behaviors — behaviors that are important in our universe and are worthy of probing deeply by both theoretical and observational means (see Ref. [2] and earlier papers by Wheeler reprinted therein and also Ref. [3]). It was obvious to Wheeler that analytical tools by themselves would not be sufficient to reveal the richness of geometrodynamics, so he encouraged his colleagues and students to begin developing numerical tools [4-6], and he encouraged Joseph Weber to develop technology for gravitational-wave observations [7].

Today, a half century later, numerical relativity has finally reached sufficient maturity (for a review, see Ref. [8] and the references therein) that, hand in hand with analytical relativity, it can be used to explore nonlinear geometrodynamics in generic situations; and gravitationalwave detectors are sufficiently mature [9-13] that they may soon observe nonlinear geometrodynamics in blackhole collisions.

Unfortunately, there is a serious obstacle to extract- 
ing geometrodynamical insights from numerical relativity simulations: a paucity of good tools for visualizing the dynamics of curved spacetime. We are reasonably sure that buried in the billions of numbers produced by numerical-relativity simulations there are major discoveries to be made, but extracting those discoveries is exceedingly difficult and perhaps impossible with the tools we have had thus far.

Until now, curved spacetime has been visualized primarily via (isometric) embedding diagrams (Sec. 23.8 of Ref. [14]): choosing spacelike two dimensional surfaces in spacetime, and embedding them in flat 3-dimensional Euclidean space or 2+1-dimensional Minkowski spacetime in a manner that preserves the surfaces' intrinsic geometry. (For some examples of embedding diagrams applied to black-hole spacetimes, see, e.g., Refs. [15-17]). Unfortunately, such embedding diagrams are of very limited value. They capture only two dimensions of spacetime, and the 2-surfaces of greatest interest often cannot be embedded globally in flat Euclidean 3-space or flat Minkowski 2+1-dimensional spacetime [15, 18-20]. Mixed Euclidean/Minkowski embeddings are often required (e.g. Fig. 4 of Ref. [15]), and such embeddings have not proved to be easily comprehended. Moreover, although it is always possible to perform a local embedding in a flat 3-space (in the vicinity of any point on the two-surface), when one tries to extend the embedding to cover the entire two-surface, one often encounters discontinuities analogous to shocks in fluid mechanics $[18,20]$.

A systematic approach to understanding the connection between nonlinear near-field dynamics in general relativity and emitted gravitational waves is being developed by Rezzolla, Jaramillo, Macedo, and Moesta [2124]. This approach focuses on correlations between data on a surface at large radius (ideally null infinity) and data on world tubes in the source region (such as black hole horizons). The purpose is to use such correlations to infer the dynamics of a black hole (e.g. the kick) directly from data on its horizon. While we find this approach exciting and attractive, in our own work we seek a more direct set of tools: tools that can probe the dynamics of spacetime curvature that cause such correlations in the first place, and that can be more readily and intuitively applied to a wider range of other geometrodynamic phenomena. It is our hope that eventually our tools and those of Rezzolla et. al. [21-23] will provide complementary pictures for understanding spacetime dynamics, and particularly black hole kicks.

We have introduced our new set of tools in a recent paper in Physical Review Letters [1]. They are tools for visualizing spacetime curvature, called tidal tendex lines, tendicities, and tendexes; and frame-drag vortex lines, vorticities and vortexes. These tools capture the full details of the Weyl curvature tensor (vacuum Riemann tensor), which embodies spacetime curvature. They do so in three dimensional, dynamically evolving pictures, of which snapshots can be printed in a paper such as this one, and movies can be made available online. ${ }^{1}$ Specifically, as of this writing two movies can be seen at Refs. [26, 27]; one shows the vortex lines from a rotating current quadrupole, the other, vortex lines from two particles that collide head on with transverse, antiparallel spins.

We have found these tools to be an extremely powerful way to visualize the output of numerical simulations. We have also used them to obtain deep new insights into old analytical spacetimes. We have applied them, thus far, to pedagogical linear-gravity problems (this paper and [28]), to stationary and perturbed black holes (Paper II in this series), and to simulations of the inspiral and mergers of spinning black holes ([1] and Paper III). We plan to apply them in the future in a variety of other geometrodynamical venues, such as black holes ripping apart neutron stars and curved spacetime near various types of singularities.

This is the first of a series of papers in which we will (i) present these tools, (ii) show how to use them, (iii) build up physical intuition into them, and (iv) employ them to extract geometrodynamical insights from numericalrelativity simulations. Specifically:

In this paper (Paper I), we introduce these vortex and tendex tools, and we then apply them to weak-gravity situations (linearized general relativity) with special focus on the roles of vortexes and tendexes in gravitationalwave generation. In a closely related paper [28], three of us have applied these tools to visualize asymptotic gravitational radiation and explore the topology of its vortex and tendex lines, and also to explore a linearized gravity model of an extreme-kick merger. In Paper II we shall apply our new tools to quiescent black holes and quasi-normal modes of black holes, with special focus once again on the roles of vortexes and tendexes in generating gravitational waves. In Paper III and subsequent papers we shall apply our tools to numerical simulations of binary black holes, focusing on nonlinear geometrodynamics in the holes' near zone and how the near-zone vortexes and tendexes generate gravitational waves.

The remainder of this paper is organized as follows:

In Sec. II A we review the well-known split of the Weyl curvature tensor into its "electric" and "magnetic" parts $\mathcal{E}_{i j}$ and $\mathcal{B}_{i j}$, and in Sec. IIB we review the Maxwelllike evolution equations for $\mathcal{E}_{i j}$ and $\mathcal{B}_{i j}$ and discuss the mathematical duality between these fields. Then in Sec. III we review the well known physical interpretation of

\footnotetext{
1 Just as there is no unique method to evolve field lines in electromagnetism, so too is there no unique way to match tendex or vortex lines at one time with others at a later time. Nevertheless, animations of field lines are useful for pedagogical purposes and for building intuition [25]. While some of the authors and colleagues are investigating how to evolve tendex and vortex lines in generic situations, the animations of the lines posted online all have special symmetries that provide a natural way to connect lines at one time with lines at the next.
} 
$\mathcal{E}_{i j}$ as the tidal field that drives geodesic deviation and the not so well known interpretation of $\mathcal{B}_{i j}[29,30]$ as the frame-drag field that drives differential frame-dragging, and we derive the equation of differential frame dragging.

In Sec. IV we introduce our new set of tools for visualizing spacetime curvature. Specifically: In Sec. IV A we introduce tendex lines and their tendicities, and we quantify them by their stretching or compressional force on a person; and we also introduce vortex lines and their vorticities and quantify them by their twisting (precessional) force on gyroscopes attached to the head and feet of a person. Then in Sec. IV B we introduce vortexes and tendexes (bundles of vortex and tendex lines that have large vorticity and tendicity) and give examples.

In the remainder of this paper we illustrate these new concepts by applying them to some well known, weakgravity, analytic examples of spacetime curvature. In Sec. V we focus on the spacetime curvature of stationary systems, and in Sec. VI we focus on dynamical systems and develop physical pictures of how they generate gravitational waves.

More specifically, in Sec. VA, we compute $\mathcal{E}_{i j}$ and $\mathcal{B}_{i j}$ for a static, gravitating, spinning point particle; we explain the relationship of $\mathcal{B}_{i j}$ to the particle's dipolar "gravitomagnetic field", we draw the particle's tendex lines and vortex lines, and we identify two vortexes that emerge from the particle, a counterclockwise vortex in its "north polar" region and a clockwise vortex in its "south polar" region. In Sec. VB, we draw the vortex lines for two spinning point particles that sit side by side with their spins in opposite directions, and we identify their four vortexes. Far from these particles, they look like a single point particle with a current quadrupole moment. In Sec. V C, we draw the vortex lines for such a currentquadrupole particle and identify their vortexes. Then in Sec. VD, we show that the tendex lines of a massquadrupole particle have precisely the same form as the vortex lines of the current-quadrupole particle, and we identify the mass quadrupole's four tendexes.

Turning to dynamical situations, in Sec. VI A we compute $\mathcal{E}_{i j}$ and $\mathcal{B}_{i j}$ for a plane gravitational wave, we express them in terms of the Weyl scalar $\Psi_{4}$, and we draw their vortex and tendex lines. In Sec. VIB we explore the quadrupolar $(l=2, m=0)$ angular pattern of gravitational waves from the head-on collision of two black holes, and we draw their vortex lines and tendex lines, intensity-coded by vorticity and tendicity, on a sphere in the wave zone. In Sec. VIC we compute $\mathcal{E}_{i j}$ and $\mathcal{B}_{i j}$ for a general, time varying current quadrupolar particle, and then in Secs. VID and VIE we specialize to a rotating current quadrupole and an oscillating current quadrupole, and draw their vortex and tendex lines. Our drawings and the mathematics reveal that the particle's outgoing gravitational waves are generated by its near-zone vortexes. The rotating current quadrupole has four vortexes that spiral outward and backward like four water streams from a rotating sprinkler. As it bends backward, each vortex acquires an accompanying ten- dex; and the vortex and tendex together become a gravitational wave crest or gravitational wave trough. The oscillating current quadrupole, by contrast, ejects vortex loops that travel outward, acquiring accompanying tendex loops with strong tendicity on the transverse segment of each loop and weak on the radial segment - thereby becoming outgoing gravitational waves.

In Sec. VIF we show that a time varying mass quadrupole produces the same phenomena as a time varying current quadrupole, but with vortexes and tendexes interchanged.

In Sec. VIG we study the vortexes and tendexes of a slow-motion binary made of nonspinning point particles. In the near zone, the tendex lines transition, as one moves radially outward, from those of two individual particles (radial and circular lines centered on each particle) toward those of a single spherical body (radial and circular lines centered on the binary and produced by the binary's mass monopole moment). In the transition zone and inner wave zone, the mass monopole continues to dominate. Then at radii $r \sim a^{2} / M$ (where $a$ is the particles' separation and $M$ is the binary's mass), the radiative quadrupole moment begins to take over and the tendex lines gradually transition into the outward-andbackward spiraling lines of a rotating quadrupole.

We make some concluding remarks in Sec. VII.

Throughout this paper we use geometrized units with $c=G=1$, and we use the sign conventions of MTW [14] for the metric signature, the Weyl curvature, and the Levi-Civita tensor. We use Greek letters for spacetime indices (0-3) and Latin letters for spatial indices (1-3), and we use arrows over 4 -vectors and bold-face font for spatial 3-vectors and for tensors. In orthonormal bases, we use hats over all kinds of indices.

\section{THE TIDAL FIELD $\mathcal{E}_{i j}$ AND FRAME-DRAG FIELD $\mathcal{B}_{i j}$}

\section{A. 3+1 split of Weyl curvature tensor into $\mathcal{E}_{i j}$ and $\mathcal{B}_{i j}$}

For a given spacetime, the Weyl curvature tensor can be calculated from the Riemann tensor by subtracting Riemann's trace from itself; i.e., by subtracting from Riemann the following combinations of the Ricci curvature tensor $R^{\mu}{ }_{\nu}$, and Ricci curvature scalar $R$ (Eq. (13.50) of MTW [14]):

$$
C_{\rho \sigma}^{\mu \nu}=R_{\rho \sigma}^{\mu \nu}-2 \delta_{[\rho}^{[\mu} R_{\sigma]}^{\nu]}+\frac{1}{3} \delta_{[\rho}^{[\mu} \delta_{\sigma]}^{\nu]} R .
$$

Here $\delta^{\mu}{ }_{\rho}$ is the Kronecker delta, and the brackets [ ] represent antisymmetrization. Note that in vacuum, $C_{\rho \sigma}^{\mu \nu}=R_{\rho \sigma}^{\mu \nu}$, and thus in vacuum the Weyl tensor contains all information about the spacetime curvature.

Let us pick a foliation of spacetime into a family of spacelike hypersurfaces. We shall denote by $u^{\mu}$ the 4 velocity of observers who move orthogonal to the folia- 
tion's space slices, and by $\gamma_{\mu \nu}=g_{\mu \nu}+u_{\mu} u_{\nu}$ the induced spatial three metric on these slices, so that $\gamma_{\alpha}{ }^{\mu}$ is the projection operator onto the slices. As is well known, e.g. [31], using this projection operator, we can split the Weyl tensor covariantly into two irreducible parts, which are symmetric, trace-free tensors that lie in the foliation's hypersurfaces (i.e. that are orthogonal to $u^{\mu}$ ). These pieces are

$$
\mathcal{E}_{\alpha \beta}=\gamma_{\alpha}{ }^{\rho} \gamma_{\beta}{ }^{\sigma} C_{\rho \mu \sigma \nu} u^{\mu} u^{\nu}, \quad \text { i.e. } \mathcal{E}_{i j}=C_{i \hat{0} j \hat{0}},
$$

an even parity field called the "electric" part of $C_{\rho \sigma}^{\mu \nu}$; and

$$
\mathcal{B}_{\alpha \beta}=-\gamma_{\alpha}{ }^{\rho} \gamma_{\beta}{ }^{\sigma *} C_{\rho \mu \sigma \nu} u^{\mu} u^{\nu}, \quad \text { i.e. } \mathcal{B}_{i j}=\frac{1}{2} \epsilon_{i p q} C_{j \hat{0}}^{p q},
$$

an odd parity field known as the "magnetic" part of $C_{\rho \sigma}^{\mu \nu}$. Here the symbol * represents the (left) Hodge dual, ${ }^{*} C_{\rho \mu \sigma \nu}=\frac{1}{2} \epsilon_{\rho \mu \eta \lambda} C^{\eta \lambda}{ }_{\sigma \nu}$; and for each field the second expression is written in $3+1$ notation: the Latin (spatial) indices are components in the foliation's hypersurface, and the $\hat{0}$ is a component on the foliation's unit time basis vector $\vec{e}_{\hat{0}} \equiv \vec{u}$. Our normalization for the Levi-Civita tensor is that of MTW: in a right-handed orthonormal frame, $\epsilon_{\hat{0} \hat{1} \hat{2} \hat{3}}=+1$, and the spatial LeviCivita tensor is defined by $\epsilon_{i p q}=\epsilon_{\hat{0} i p q}$, with $\epsilon_{\hat{1} \hat{2} \hat{3}}=1$ in a right-handed orthonormal basis. Note that Eqs. (2.2) are a direct and intentional analogy to the decomposition of the Maxwell tensor of electromagnetism $F_{\mu \nu}$ into the familiar electric and magnetic fields $E_{i}$ and $B_{i}[31]$ :

$$
E_{i}=F_{i \hat{0}}, \quad B_{i}=-{ }^{*} F_{i \hat{0}}=\frac{1}{2} \epsilon_{i p q} F^{p q} .
$$

Note that our sign conventions differ from [31], where $\epsilon_{\hat{0} \hat{1} \hat{2} \hat{3}}=-1$, and so Eq. (2.2b) has an additional minus sign in order to maintain a strict analogy with the magnetic field $B_{i}$ of electromagnetism. This results in a $\mathcal{B}_{i j}$ defined with a different sign convention than, for example, in $[32,33]$.

\section{B. Evolution of $\mathcal{E}_{i j}$ and $\mathcal{B}_{i j}$}

The propagation equations for the Weyl tensor and its gravito-electromagnetic representation are the Bianchi identities. We shall write them down and discuss them in three contexts: a general foliation and coordinate system, the local Lorentz frame of a freely falling observer, and the weak-gravity, nearly Minkowski spacetimes of the current paper (Paper I in this series).

\section{General foliation and coordinate system in the language of numerical relativity}

Because this paper is a foundation for using $\mathcal{E}_{i j}$ and $\mathcal{B}_{i j}$ to interpret the results of numerical relativity simulations, we shall write their evolution equations (the
Bianchi identities) in a general coordinate system of the type used in numerical relativity, and we shall discuss these equations' mathematical structure in the language of numerical relativity.

We denote by $t$ a time coordinate that is constant on the foliation's hypersurfaces, and by $\alpha$ and $\vec{\beta}$ the foliation's lapse and shift functions, so the orthogonal observers' 4 -velocity is $\vec{u}=\alpha^{-1}\left(\vec{\partial}_{t}-\vec{\beta}\right)$. The $3+1$ split divides the Bianchi identities into evolution equations that govern the time evolution of the spatial fields, and constraint equations that are obeyed by the fields on each time slice. The evolution equations are [34, 35]

$$
\begin{aligned}
\partial_{t} \mathcal{E}_{i j}= & \mathcal{L}_{\beta} \mathcal{E}_{i j}+\alpha\left[D_{k} \mathcal{B}_{l(i} \epsilon_{j)}^{k l}-3 \mathcal{E}_{(i}^{k} K_{j) k}\right. \\
& \left.+K_{k}^{k} \mathcal{E}_{i j}-\epsilon_{i}{ }^{k l} \mathcal{E}_{k m} K_{l n} \epsilon_{j}{ }^{m n}+2 a_{k} \mathcal{B}_{l(i} \epsilon_{j)}{ }^{k l}\right] \\
\partial_{t} \mathcal{B}_{i j}= & \mathcal{L}_{\beta} \mathcal{B}_{i j}+\alpha\left[-D_{k} \mathcal{E}_{l(i} \epsilon_{j)}^{k l}-3 \mathcal{B}^{k}{ }_{(i} K_{j) k}\right. \\
& \left.+K^{k}{ }_{k} \mathcal{B}_{i j}-\epsilon_{i}{ }^{k l} \mathcal{B}_{k m} K_{l n} \epsilon_{j}{ }^{m n}-2 a_{k} \mathcal{E}_{l(i} \epsilon_{j)}^{k l}\right] .
\end{aligned}
$$

Here the extrinsic curvature, Lie derivative on a second rank tensor, and acceleration of the slicing are respectively defined by

$$
\begin{aligned}
K_{i j} & =-\frac{1}{2 \alpha}\left(\partial_{t} \gamma_{i j}-D_{i} \beta_{j}-D_{j} \beta_{i}\right) \\
\mathcal{L}_{\beta} \mathcal{E}_{i j} & =\beta^{k} D_{k} \mathcal{E}_{i j}+\mathcal{E}_{i k} D_{j} \beta^{k}+\mathcal{E}_{k j} D_{i} \beta^{k} \\
a_{k} & =D_{k} \ln \alpha .
\end{aligned}
$$

The derivative $D_{i}$ is the covariant derivative associated with the induced metric $\gamma_{i j}$ on the slices. The evolution system (2.4) is closed by an additional evolution equation for the 3-metric, which is Eq. (2.5), and evolution equations for the extrinsic curvature and the 3-dimensional connection $\Gamma_{i j}^{k}$, which are

$$
\begin{aligned}
\partial_{t} K_{i j}= & \mathcal{L}_{\beta} K_{i j}-\alpha\left[\partial_{k} \Gamma_{i j}^{k}-\Gamma_{l j}^{k} \Gamma_{k i}^{l}+\partial_{i} \partial_{j} q\right. \\
& \left.+\partial_{i} \ln \alpha \partial_{j} \ln \alpha-\Gamma_{i j}^{k} \partial_{k} q-2 \mathcal{E}_{i j}+K K_{i j}\right] \\
\partial_{t} \Gamma_{i j}^{k}= & \mathcal{L}_{\beta} \Gamma_{i j}^{k}-\alpha D^{k} K_{i j}+K_{i j} D^{k} \alpha-2 K_{(i}^{k} D_{j)} \alpha \\
& +2 \alpha \epsilon^{k l}{ }_{(i} \mathcal{B}_{j) l},
\end{aligned}
$$

where we have defined

$$
\begin{aligned}
q= & \ln \left(\alpha \gamma^{-1 / 2}\right), \\
\mathcal{L}_{\beta} \Gamma_{i j}^{k}= & \beta^{l} \partial_{l} \Gamma_{i j}^{k}+2 \Gamma_{l(j}^{k} \partial_{i)} \beta^{l} \\
& -\Gamma_{i j}^{l} \partial_{l} \beta^{k}+\partial_{i} \partial_{j} \beta^{k} .
\end{aligned}
$$

The above equations are symmetric hyperbolic if $q$ and $\beta^{i}$ are specified functions of time and space.

The constraint equations on each slice are the definitions of $\mathcal{E}_{i j}$ and $\mathcal{B}_{i j}$,

$$
\begin{aligned}
& \mathcal{E}_{i j}={ }^{(3)} R_{i j}+K K_{i j}-K_{i}^{k} K_{j k}, \\
& \mathcal{B}_{i j}=\epsilon_{j}{ }^{l k} D_{k} K_{l i}
\end{aligned}
$$


from which the Einstein constraints follow from the condition that $\mathcal{E}_{i j}$ and $\mathcal{B}_{i j}$ are symmetric and tracefree; and the definition of $\Gamma_{i j}^{k}$,

$$
\Gamma_{i j}^{k}=\frac{1}{2} \gamma^{k \ell}\left(\partial_{i} \gamma_{j \ell}+\partial_{j} \gamma_{i \ell}-\partial_{\ell} \gamma_{i j}\right)
$$

The Bianchi identities imply derivative constraints on $\mathcal{E}_{i j}$ and $\mathcal{B}_{i j}$ :

$$
\begin{aligned}
& D^{i} \mathcal{E}_{i j}=\mathcal{B}_{i k} K^{i}{ }_{l} \epsilon^{k l}{ }_{j}, \\
& D^{i} \mathcal{B}_{i j}=-\mathcal{E}_{i k} K^{i}{ }_{l} \epsilon^{k l}{ }_{j} .
\end{aligned}
$$

These last equations are automatically satisfied if Eqs. (2.11) are satisfied. Eqs. (2.13) are nonlinear, but otherwise they have the same structure as the constraints in simple electromagnetism.

Note also that the equations governing $\mathcal{E}$ and $\mathcal{B}$, Eqs (2.4) and (2.13) share another similarity with the field equations of electromagnetism. Namely, just as the Maxwell equations are invariant under the duality transformation

$$
E \rightarrow B, \quad B \rightarrow-E,
$$

i.e. under a rotation in the complexified notation

$$
\boldsymbol{E}-i \boldsymbol{B} \rightarrow e^{i \pi / 2}(\boldsymbol{E}-i \boldsymbol{B}),
$$

so the exact Maxwell-like Bianchi identities (2.4) are also invariant under the same duality transformation

$$
\mathcal{E} \rightarrow \mathcal{B}, \quad \mathcal{B} \rightarrow-\mathcal{E} .
$$

This duality in the structure of Eqs. (2.4) and also (2.13) does not in general enable one to construct one metric solution of Einstein's equations from another, known solution. However, as we shall see, we can utilize this duality in weakly gravitating systems to find the $\mathcal{E}$ and $\mathcal{B}$ generated by one set of source moments, given the expressions for $\mathcal{E}$ and $\mathcal{B}$ for a dual set of moments.

\section{Local Lorentz frame of a freely falling observer}

When one introduces the local Lorentz frame of a freely falling observer in curved spacetime, one necessarily specializes one's foliation: (i) The local-Lorentz foliation's space slices are flat at first order in distance from the observer's world line, so its extrinsic curvature $K_{i j}$ vanishes along the observer's world line. (ii) Because the observer is freely falling, her acceleration $a_{k}$ vanishes, which means that successive hypersurfaces in the foliation are parallel to each other along the observer's world line.

These specializations, plus the vanishing shift $\beta_{i}=0$ and unit lapse function $\alpha=1$ of a local Lorentz frame, bring the constraint and evolution equations (2.13) and (2.4) into the following Maxwell-like form:

$$
\begin{aligned}
\boldsymbol{\nabla} \cdot \mathcal{E} & =0, \quad \boldsymbol{\nabla} \cdot \mathcal{B}=0 \\
\frac{\partial \mathcal{E}}{\partial t}-(\boldsymbol{\nabla} \times \mathcal{B})^{S} & =0, \quad \frac{\partial \mathcal{B}}{\partial t}+(\boldsymbol{\nabla} \times \mathcal{E})^{S}=0 .
\end{aligned}
$$

Here the superscript $S$ means "take the symmetric part" and the remaining notation is the same as in the flatspacetime Maxwell equations (including changing from D to $\boldsymbol{\nabla}$ for the spatial gradient).

\section{Weak-gravity, nearly Minkowski spacetimes}

In this paper's applications (Secs. V and VI), we shall specialize to spacetimes and coordinate systems that are weakly perturbed from Minkowski, and we shall linearize in the perturbations. In this case, the Bianchi identities (2.4) take on precisely the same Maxwell-like form as in a local Lorentz frame in strongly curved spacetime, Eqs. (2.15). To see that this is so, note that $\beta_{k}, K_{j k}, a_{k}, \mathcal{E}_{j k}$, and $\mathcal{B}_{j k}$ are all first order perturbations and that $\alpha$ is one plus a first-order perturbation; and linearize Eqs. (2.4) in these first order quantities.

When the weak-gravity spacetime is also characterized by slow motion, so its source regions are small compared to the wavelengths of its gravitational waves, the evolution equations control how the near-zone $\mathcal{E}_{j k}$ and $\mathcal{B}_{j k}$ get transformed into gravitational wave fields. For insight into this, we specialize to harmonic gauge, in which the trace-reversed metric perturbation $\bar{h}_{\mu \nu}$ is divergence-free, $\partial^{\mu} \bar{h}_{\mu \nu}=0$.

Then in the near zone, $\mathcal{E}_{j k}$ and $\mathcal{B}_{j k}$ [which are divergence free and curl free by Eqs. (2.15)] are expressible in terms of the metric perturbation itself as

$$
\mathcal{E}_{i j}=-\frac{1}{2} \partial_{i} \partial_{j} h_{00}, \quad \mathcal{B}_{i j}=\frac{1}{2} \epsilon_{i}^{p q} \partial_{q} \partial_{j} h_{p 0} .
$$

Because $h_{00}$, at leading order in $r / \lambda$ (ratio of radius to reduced wavelength) contains only mass multipole moments (Eq. (8.13a) of [36]), so also $\mathcal{E}_{j k}$ contains only mass multipole moments. And because $h_{p 0}$ at leading order in $r / \lambda$ contains only current multipole moments, so also $\mathcal{B}_{j k}$ contains only current multipole moments.

In the wave zone, by contrast, Eqs. (2.15) show that the locally plane waves are sustained by mutual induction between $\mathcal{E}$ and $\mathcal{B}$, just like for electromagnetic waves, which means that these two wave-zone fields must contain the same information. This is confirmed by the wave-zone expressions for $\mathcal{E}_{j k}$ and $\mathcal{B}_{j k}$ in terms of the metric perturbation,

$$
\mathcal{E}_{i j}=-\frac{1}{2} \partial_{0}^{2} h_{i j}, \quad \mathcal{B}_{i j}=-\frac{1}{2} \epsilon_{i}^{p q} n_{p} \partial_{0}^{2} h_{q j} .
$$

Both fields are expressed in terms of the same quantity, $h_{i j}$. In addition, in the wave zone, $\mathcal{E}$ and $\mathcal{B}$ are related to each other through a $\pi / 4$ rotation of their polarization tensors (see Section VIA below). Correspondingly, we will see in Section VI that, if a time-varying mass moment produces + polarized radiation in the wave zone, then the current moment that is dual to it produces $\times$ polarized radiation of the same magnitude.

In the transition zone, the inductive coupling between $\mathcal{E}$ and $\mathcal{B}$, embodied in Eqs. (2.4), enables these equations 
to act like a blender, mixing up the multipolar information that in the near zone is stored separately in these two fields. After an infinite amount of inductive blending, we arrive at future null infinity, $\mathcal{I}^{+}$, where the mixing has been so thorough that $\mathcal{E}$ and $\mathcal{B}$ contain precisely the same information, though it is distributed differently among their tensor components [Eqs. (2.17)].

The details of this transition-zone mixing, as embodied in Eqs. (2.15), are in some sense the essence of gravitational wave generation. We shall explore those details visually in Sec. VI by tracking the tendex and vortex lines (introduced in Sec IV) that extend from the near zone, through the transition zone, and into the far zone.

Finally, note that the duality of $\mathcal{E}$ and $\mathcal{B}$ becomes especially convenient for slow-motion systems, where we can relate $\mathcal{E}$ and $\mathcal{B}$ to source multipole moments that appear in the weak-field near zone. In particular: to obtain the $\mathcal{E}$ and $\mathcal{B}$ generated by a specific current moment $\mathcal{S}_{\ell}$, we can simply apply the duality transformation (2.14) to the $\mathcal{E}$ and $\mathcal{B}$ for its dual moment, which is the mass moment $\mathcal{I}_{\ell}$, but with one caveat: The differing normalizations used for mass moments and current moments [36] enforce the duality relation

$$
\mathcal{I}_{\ell} \rightarrow \frac{2 \ell}{\ell+1} \mathcal{S}_{\ell}, \quad \mathcal{S}_{\ell} \rightarrow-\frac{\ell+1}{2 \ell} \mathcal{I}_{\ell}
$$

when making this duality transformation; note that both transformations, Eqs. (2.14) and (2.18), must be made at once to arrive at the correct expressions; see Sec. VI.

\section{PHYSICAL INTERPRETATIONS OF $\mathcal{E}_{i j}$ AND $\mathcal{B}_{i j}$}

It is rather well known that in vacuum ${ }^{2}$ the electric part of the Weyl tensor, $\mathcal{E}_{i j}$, describes tidal gravitational accelerations: the relative acceleration of two freely falling particles with separation vector $\xi^{k}$ is $\Delta a^{i}=$ $\mathcal{E}^{i}{ }_{j} \xi^{j}$. For this reason $\mathcal{E}_{i j}$ is often called the tidal field, a name that we shall adopt.

Not so well known is the role of the magnetic part of the Weyl tensor $\mathcal{B}_{j k}$ as governing differential frame dragging, i.e. the differential precession of inertial reference frames: in vacuum ${ }^{2}$ a gyroscope at the tip of the separation vector $\xi^{k}$, as observed in the local Lorentz frame of an observer at the tail of $\xi^{k}$, precesses with angular velocity $\Delta \Omega^{j}=$ $\mathcal{B}^{j}{ }_{k} \xi^{k}$. For this reason, we call $\mathcal{B}_{j k}$ the frame-drag field.

2 In a non-vacuum region of spacetime, the local stress-energy tensor also contributes to tidal accelerations via its algebraic relation to the Ricci tensor which in turn contributes to the Riemann tensor. In this case, $\mathcal{E}_{i j}$ describes that portion of the tidal acceleration due to the "free gravitational field", i.e. the portion that is sourced away from the location where the tidal acceleration is measured; and similarly for $\mathcal{B}_{j k}$ and differential frame dragging. In this paper we shall ignore this subtle point and focus on tidal forces and differential frame-dragging in vacuum.

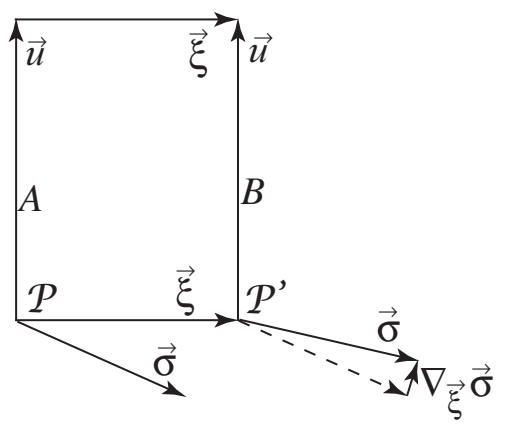

FIG. 1: Spacetime geometry for computing the precession of a gyroscope at one location $\mathcal{P}^{\prime}$, relative to gyroscopic standards at a nearby location $\mathcal{P}$.

We deduced this frame-drag role of $\mathcal{B}_{j k}$ during our research and then searched in vain for any reference to it in the literature, while writing our Physical Review Letter on vortexes and tendexes [1]. More recently we have learned that this role of $\mathcal{B}_{j k}$ was known to Frank Estabrook and Hugo Wahlquist [29] 46 years ago and was rediscovered two years ago by Christoph Schmidt [30] (who states it without proof).

For completeness, in this section we shall give a precise statement and proof of the frame-drag role of $\mathcal{B}_{j k}$, and a corresponding precise statement of the tidal-acceleration role of $\mathcal{E}_{j k}$.

\section{A. Physical setup}

Consider an event $\mathcal{P}$ in spacetime and an observer labeled $A$ whose world line passes through $\mathcal{P}$ and has 4 velocity $\vec{u}$ there; see Fig. 1 . Introduce an infinitesimally short 4 -vector $\vec{\xi}$ at $\mathcal{P}$, that is orthogonal to $\vec{u}$ and thus is seen as spatial by observer $A$. Denote by $\mathcal{P}^{\prime}$ the event at the tip of $\vec{\xi}$. Introduce a second observer $B$ whose world line passes through $\mathcal{P}^{\prime}$ and is parallel there to the world line of observer $A$, so if we denote $B$ 's 4 -velocity by the same symbol $\vec{u}$ as that of $A$ and imagine a vector field $\vec{u}$ that varies smoothly between the two world lines, then $\nabla_{\vec{\xi}} \vec{u}=0$ at $\mathcal{P}$. Let $\vec{\xi}$ be transported by observer $A$ in such a way that it continues to reach from world line $A$ to world line $B$. Then the vectors $\vec{u}$ and $\vec{\xi}$ satisfy the following three relations at $\mathcal{P}$ :

$$
\vec{\xi} \cdot \vec{u}=0, \quad[\vec{u}, \vec{\xi}]=0, \quad \nabla_{\vec{\xi}} \vec{u}=0 .
$$

The first says that the separation vector is purely spatial at $\mathcal{P}$ in the reference frame of observer $A$; the second says that $\vec{\xi}$ continues to reach between world lines $A$ and $B$, so the quadrilateral formed by $\vec{u}$ and $\vec{\xi}$ in Fig. 1 is closed; the third says that the two observers' world lines are parallel to each other at $\mathcal{P}$-i.e., these observers regard themselves as at rest with respect to each other. 


\section{B. Interpretation of $\mathcal{E}_{i j}$ as the tidal field}

Let the two observers $A$ and $B$ fall freely, i.e. move on geodesics. Then for this physical setup, the equation of geodesic deviation states that [e.g. [14] Eq. (11.10)]

$$
\nabla_{\vec{u}} \nabla_{\vec{u}} \vec{\xi}=-\mathrm{R}(\ldots, \vec{u}, \vec{\xi}, \vec{u})
$$

where $\mathrm{R}$ is the Riemann tensor. In physical language, the left side is the acceleration $\Delta \vec{a}$ of observer $B$ at $\mathcal{P}^{\prime}$, as measured in the local Lorentz frame of observer $A$ at $\mathcal{P}$. This relative acceleration is purely spatial as seen by observer $A$, and the right side of Eq. (3.2) tells us that in spatial, 3 -dimensional vector and tensor notation (and in vacuum so $R_{\alpha \beta \gamma \delta}=C_{\alpha \beta \gamma \delta}$ ), it is given by

$$
\Delta a^{j}=-R^{j}{ }_{\hat{0} k \hat{0}} \xi^{k}=-\mathcal{E}^{j}{ }_{k} \xi^{k} ; \text { i.e. } \Delta \boldsymbol{a}=-\mathcal{E}\left({ }_{\boldsymbol{C}}, \boldsymbol{\xi}\right) \text {. }
$$

Since (as is well known) this relative acceleration produces the Earth's tides when $\mathcal{E}_{j k}$ is caused by the moon and sun, $\mathcal{E}_{j k}$ is called the tidal field, and Eq. (3.3) is known as the tidal acceleration equation.

\section{Interpretation of $\mathcal{B}_{i j}$ as the frame-drag field}

Next let the two observers $A$ and $B$ in Fig. 1 be accelerated if they wish (with the same 4-acceleration $\vec{a}$ up to differences proportional to $\vec{\xi}$ ), and give each of them a spatial unit vector $\vec{\sigma}$ that is tied to an inertial-guidance gyroscope, so the following relations are satisfied:

$$
\vec{\sigma} \cdot \vec{u}=0, \quad \vec{\sigma} \cdot \vec{\sigma}=1, \quad \nabla_{\vec{u}} \vec{\sigma}=(\vec{a} \cdot \vec{\sigma}) \vec{u}, \quad \vec{a} \equiv \nabla_{\vec{u}} \vec{u} .
$$

The first of these says that $\vec{\sigma}$ is purely spatial as seen in the observer's reference frame; the second says that $\vec{\sigma}$ has unit length; the third is the Fermi-Walker transport law for an inertial-guidance gyroscope.

The local-frame-dragging-induced rate of change of $\vec{\sigma}$ at $\mathcal{P}^{\prime}$, as measured using inertial-direction standards at $\mathcal{P}$, is $\nabla_{\vec{u}} \nabla_{\vec{\xi}} \vec{\sigma}$. We can write this as

$$
\begin{aligned}
\nabla_{\vec{u}} \nabla_{\vec{\xi}} \vec{\sigma} & =\nabla_{\vec{\xi}} \nabla_{\vec{u}} \vec{\sigma}+\left[\nabla_{\vec{u}}, \nabla_{\vec{\xi}}\right] \vec{\sigma} \\
& \left.=\nabla_{\vec{\xi}} \nabla_{\vec{u}} \vec{\sigma}+\mathrm{R}_{(}, \vec{\sigma}, \vec{u}, \vec{\xi}\right)
\end{aligned}
$$

where $\mathrm{R}$ is the Riemann tensor and we have used the fact that $[\vec{u}, \vec{\xi}]=0$; cf. Eqs. (11.8) and (11.9) of MTW [14].

Evaluating the first term $\nabla_{\vec{\xi}} \nabla_{\vec{u}} \vec{\sigma}$ using the FermiWalker transport law [the third of Eqs. (3.4)] and the fact that the observers are momentarily at rest with respect to each other [the third of Eqs. (3.1)], we bring Eq. (3.5) into the form

$$
\nabla_{\vec{u}} \nabla_{\vec{\xi}} \vec{\sigma}=\mathrm{R}\left({ }_{-}, \vec{\sigma}, \vec{u}, \vec{\xi}\right)+\vec{u} \nabla_{\vec{\xi}}(\vec{a} \cdot \vec{\sigma})
$$

We are only interested in the spatial part of this rate of change, so we can ignore the second term on the right side of the equation. We switch to the 3-dimensional viewpoint of the observer at $\mathcal{P}$ (where our calculation is being done) and we denote the spatial part of $\nabla_{\vec{u}} \nabla_{\vec{\xi}} \vec{\sigma}$ by $\dot{\sigma}$ :

$$
\dot{\boldsymbol{\sigma}} \equiv\left[\nabla_{\vec{u}} \nabla_{\vec{\xi}} \vec{\sigma}\right]_{\text {project orthogonal to } \vec{u}} \cdot
$$

Eq. (3.6) tells us that this rate of change is not only orthogonal to $\vec{u}$ (spatial) but also orthogonal to $\sigma$; it therefore can be written as a rotation

$$
\dot{\sigma}=\Delta \Omega \times \sigma
$$

Here $\Delta \boldsymbol{\Omega}$ is the frame-dragging angular velocity at $\mathcal{P}^{\prime}$ as measured using inertial standards at $\mathcal{P}$. We can solve for this angular velocity $\Delta \boldsymbol{\Omega}$ by crossing $\boldsymbol{\sigma}$ into Eq. (3.8) and using $\boldsymbol{\sigma} \cdot \boldsymbol{\sigma}=1$ :

$$
\Delta \Omega=\sigma \times \dot{\sigma}
$$

Inserting expression (3.6) for $\dot{\boldsymbol{\sigma}}$ and switching to index notation, we obtain

$$
\Delta \Omega_{i}=\epsilon_{i j k} \sigma^{j} R_{p \hat{0} q}^{k} \sigma^{p} \xi^{q} .
$$

Rewriting the Riemann tensor component in terms of the gravitomagnetic part of the Weyl tensor (in vacuum), $R^{k}{ }_{p \hat{q} q}=-\epsilon^{k}{ }_{p s} \mathcal{B}^{s}{ }_{q}$, performing some tensor manipulations, and noticing that because $\Delta \boldsymbol{\Omega}$ is crossed into $\boldsymbol{\sigma}$ when computing the precession, any piece of $\Delta \boldsymbol{\Omega}$ along $\boldsymbol{\sigma}$ is irrelevant, we obtain

$$
\Delta \Omega_{i}=\mathcal{B}_{i j} \xi^{j}, \quad \text { i.e. } \Delta \boldsymbol{\Omega}=\mathcal{B}(\ldots, \boldsymbol{\xi}) .
$$

In words: in vacuum the frame-dragging angular velocity at $\mathcal{P}^{\prime}$, as measured using inertial directions at the adjacent event $\mathcal{P}$, is obtained by inserting the vector $\boldsymbol{\xi}$ (which reaches from $\mathcal{P}$ to $\mathcal{P}^{\prime}$ ) into one slot of the gravitomagnetic part of the Weyl tensor.

Because of the role of $\mathcal{B}_{i j}$ in this equation of differential frame dragging, we call $\mathcal{B}_{i j}$ the frame-drag field.

\section{OUR NEW TOOLS: TENDEX AND VORTEX LINES; THEIR TENDICITIES AND VORTICITIES; TENDEXES AND VORTEXES}

\section{A. Tendex lines and their tendicities; vortex lines and their vorticities}

As symmetric, trace free tensors, the tidal field $\mathcal{E}$ and frame-drag field $\mathcal{B}$ can each be characterized completely by its three principal axes (eigendirections) and its three associated eigenvalues.

If $\boldsymbol{p}$ is a (smoothly changing) unit eigenvector of the tidal field $\mathcal{E}$ (or of the frame-drag field $\mathcal{B}$ ), then the integral curves of $\boldsymbol{p}$ can be regarded as "field lines" associated with $\mathcal{E}($ or $\mathcal{B})$. For $\mathcal{E}$ we call these integral curves tidal tendex lines, or simply tendex lines ${ }^{3}$, because $\mathcal{E}$ tidally

\footnotetext{
3 The word tendex was coined by David Nichols.
} 


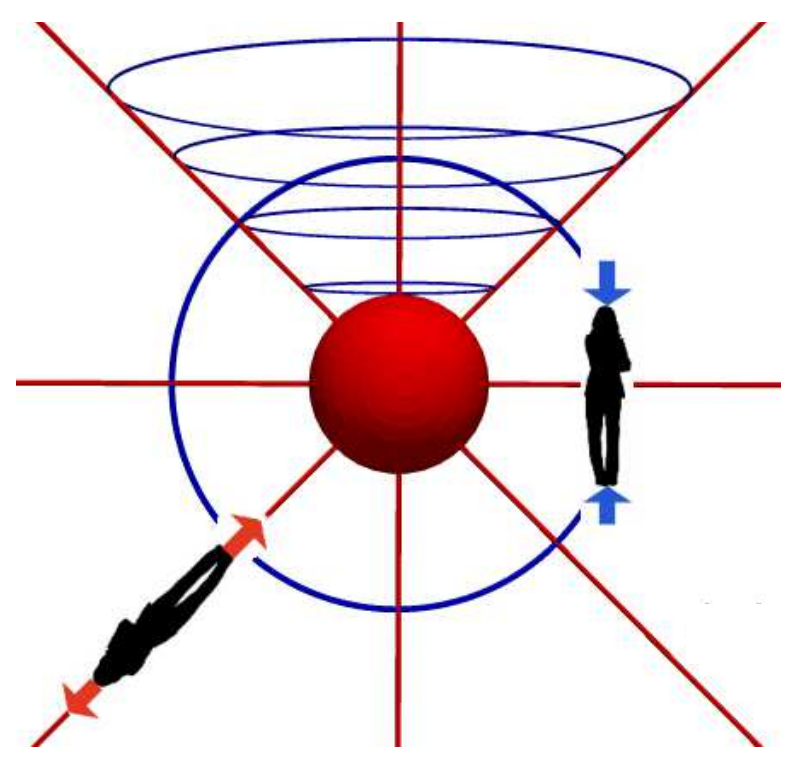

FIG. 2: (color online). Tendex lines outside a spherically symmetric, gravitating body. The lines are colored by the sign of their tendicity: red lines have negative tendicity (they stretch a person oriented along them); blue lines have positive tendicity (they squeeze).

stretches objects it encounters, and the Latin word tendere means "to stretch". For $\mathcal{B}$ we call the integral curves frame-drag vortex lines, or simply vortex lines, because $\mathcal{B}$ rotates gyroscopes, and the Latin word vertere means "to rotate". At each point $\mathcal{P}$ in space there are three orthogonal eigendirections of $\mathcal{E}$ (and three of $\mathcal{B}$ ), so through each point there pass three orthogonal tendex lines and three orthogonal vortex lines.

Outside a spherically symmetric gravitating body with mass $M$, such as the Earth or a Schwarzschild black hole, the tidal field, in a spherical polar orthonormal basis, has components

$$
\mathcal{E}_{\hat{r} \hat{r}}=-\frac{2 M}{r^{3}}, \quad \mathcal{E}_{\hat{\theta} \hat{\theta}}=\mathcal{E}_{\hat{\phi} \hat{\phi}}=+\frac{M}{r^{3}}
$$

(e.g. Sec. 1.6 and Eq. (31.4) of [14]). The tidal acceleration equation $\Delta a^{j}=-\mathcal{E}^{j}{ }_{k} \xi^{k}$ tells us that this tidal field stretches objects radially and squeezes them equally strongly in all tangential directions (see the people in Fig. 2). Correspondingly, one eigenvector of $\mathcal{E}$ is radial, and the other two are tangential with degenerate eigenvalues. This means that one set of tendex lines is radial (the red tendex lines in Fig. 2), and any curve lying on a sphere around the body is a tendex line. If we break the tangential degeneracy by picking our tangential unit eigenvectors to be the basis vectors $\boldsymbol{e}_{\hat{\theta}}$ and $\boldsymbol{e}_{\hat{\phi}}$ of a spherical polar coordinate system, then the tangential tendex lines are those vectors' integral curves - the blue curves in Fig. 2.

When the spherical body is weakly gravitating and is set rotating slowly, then it acquires a nonzero frame-drag field given by Eqs. (5.11) below. The corresponding vor-

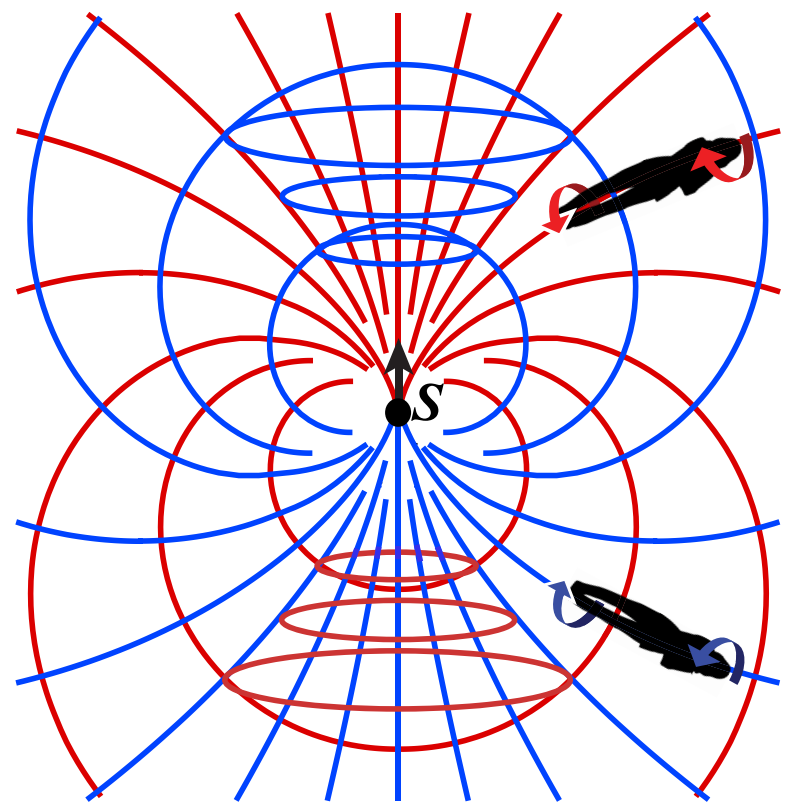

FIG. 3: (color online). Vortex lines outside a slowly spinning, spherically symmetric, gravitating body with spin angular momentum $\boldsymbol{S}$. The lines are colored by the sign of their vorticity: red lines have negative vorticity (they produce a counterclockwise differential precession of gyroscopes); blue lines have positive vorticity (clockwise differential precession).

tex lines are shown in Fig. 3. (See Sec. V A below for details.)

To any tendex (or vortex) line, with unit eigenvector $\boldsymbol{p}$, there is associated an eigenvalue $\mathcal{E}_{p p}=\mathcal{E}_{j k} p^{j} p^{k}$ which is called the line's tendicity (or $\mathcal{B}_{p p}=\mathcal{B}_{j k} p^{j} p^{k}$ which is called the line's vorticity). The physical meaning of this tendicity (or vorticity) can be read off the tidal acceleration equation (3.3) [or the equation of differential frame dragging (3.11)]. Specifically, if a person's body (with length $\ell$ ) is oriented along a tidal tendex line (Fig. 2), she feels a head-to-foot stretching acceleration $\Delta a=-\mathcal{E}_{p p} \ell$. If the line's tendicity $\mathcal{E}_{p p}$ is negative (red tendex line), her body gets stretched; if the tendicity is positive (blue tendex line), she gets compressed.

If her body is oriented along a vortex line (Fig. 3), then a gyroscope at her feet precesses around the vortex line with an angular speed, relative to inertial frames at her head, given by $\Delta \Omega=\mathcal{B}_{p p} \ell$. If the line's vorticity is negative (red vortex lines in Fig. 3), then the gyroscope at her feet precesses counterclockwise relative to inertial frames at her head, and (because $\mathcal{B}_{p p}$ is unchanged when one reverses the direction $\boldsymbol{p}$ ), a gyroscope at her head precesses counterclockwise relative to inertial frames at her feet. Correspondingly, we call the (red) vortex line a counterclockwise vortex line. If the line's vorticity is positive (blue vortex lines in Fig. 3), the precessions are clockwise and the vortex line is said to be clockwise.

For any spacetime, the tendex lines color coded by their tendicities (e.g. Fig. 2) and the vortex lines color coded by their vorticities (e.g. Fig. 3) depict visually all 
details of the Weyl curvature tensor.

Since $\mathcal{E}$ and $\mathcal{B}$ are trace free, at any point in space the sum of the three tendex lines' tendicities vanishes, and the sum of the three vorticities vanishes. Because $\mathcal{E}$ and $\mathcal{B}$ are also symmetric, each is characterized by five numbers at any point in space. The direction of one tendex line fixes two numbers and its tendicity fixes a third, leaving only two numbers to be specified. The direction of a second tendex line, in the plane orthogonal to the first, fixes a fourth number and the second line's tendicity fixes the fifth and final number - leaving the last line's direction and tendicity fully determined. Similarly for vortex lines and their vorticities.

\section{B. Vortexes and tendexes}

We give the name frame-drag vortex, or simply vortex, to a bundle of vortex lines with large vorticity. In Fig. 3 , the red vortex lines near the north polar axis, that are enclosed by blue circles, constitute a negative-vorticity (counterclockwise) vortex; the blue vortex lines near the south polar axis, that are enclosed by red circles, constitute a positive-vorticity (clockwise) vortex. These two vortexes emerge from the north and south poles of the spinning point particle.

Similarly, we give the name tidal tendex, or simply tendex, to a strong concentration of tendex lines. We shall meet our first example at the end of Sec. VD below.

\section{WEAK-GRAVITY, STATIONARY SYSTEMS}

\section{A. One stationary, weakly gravitating, spinning body}

When gravity is weak and slowly changing (e.g., outside a slowly precessing, spinning, weakly gravitating body such as the Earth), one can write the spacetime metric in the form

$$
d s^{2}=-\alpha^{2} d t^{2}+\delta_{j k}\left(d x^{j}+\beta^{j} d t\right)\left(d x^{k}+\beta^{k} d t\right)
$$

(e.g. Sec. 23.9.3 of [37]; or Chap. 10 of MTW [14] with the spatial coordinates changed slightly). Here

$$
\alpha^{2}=\left(1-\frac{2 M}{r}\right), \quad \boldsymbol{\beta}=-\frac{2 \boldsymbol{S}}{r^{2}} \times \boldsymbol{n},
$$

are the squared lapse function and the shift function, $M$ is the body's mass, $\boldsymbol{S}$ is its spin angular momentum, and

$$
r=\sqrt{x^{2}+y^{2}+z^{2}}, \quad \boldsymbol{n}=\boldsymbol{e}_{\hat{r}}
$$

are radius and the unit radial vector, with $\left\{x^{1}, x^{2}, x^{3}\right\}=$ $\{x, y, z\}$. In spherical polar coordinates (associated with the Cartesian coordinates $\{x, y, z\}$ in the usual way), the metric (5.1a) becomes

$$
\begin{aligned}
d s^{2} & =-\alpha^{2} d t^{2}+d r^{2}+r^{2} d \theta^{2}+r^{2} \sin ^{2} \theta(d \phi-\omega d t)^{2}, \\
\omega & =2 S / r^{3} .
\end{aligned}
$$

It is conventional to rewrite general relativity, in this weak-field, slow-motion situation, as a field theory in flat spacetime. In this language, the geodesic equation for a test particle takes the form

$$
d^{2} \boldsymbol{x} / d t^{2}=\boldsymbol{g}+\boldsymbol{v} \times \boldsymbol{H},
$$

which resembles the Lorentz force law in electromagnetic theory; see, e.g., [38] and references therein, especially [39]. Here $\boldsymbol{v}=d \boldsymbol{x} / d t$ is the particle's velocity [Cartesian components $(d x / d t, d y / d t, d z / d t)]$ and

$$
\begin{aligned}
\boldsymbol{g} & =-\frac{1}{2} \boldsymbol{\nabla} \alpha^{2}=-\frac{M}{r^{2}} \boldsymbol{n} \\
\boldsymbol{H} & =\boldsymbol{\nabla} \times \boldsymbol{\beta}=2\left[\frac{\boldsymbol{S}-3(\boldsymbol{S} \cdot \boldsymbol{n}) \boldsymbol{n}}{r^{3}}\right]
\end{aligned}
$$

are the body's gravitoelectric field (same as Newtonian gravitational acceleration) and its gravitomagnetic field. Note that these fields have the same monopole and dipole structures as the electric and magnetic fields of a spinning, charged particle.

In this paper we shall adopt an alternative to this "gravito-electromagnetic" viewpoint. For the gravitational influence of the mass $M$, we shall return to the Newtonian viewpoint of a gravitational acceleration $\boldsymbol{g}$ and its gradient, the tidal gravitational field ("electric" part of the Weyl tensor)

$$
\mathcal{E}=-\nabla \boldsymbol{g}, \quad \text { i.e., } \mathcal{E}_{i j}=-g_{i, j}=\Phi_{, i j}=\frac{1}{2} \alpha^{2}{ }_{, i j} .
$$

Here the comma denotes partial derivative (actually, the gradient in our Cartesian coordinate system) and $\Phi$ is the Newtonian gravitational potential, which is related to the lapse function by $\alpha^{2}=1+2 \Phi$ in the Newtonian limit. The components of this tidal field in the spherical coordinates' orthonormal basis $\boldsymbol{e}_{\hat{r}}=\partial / \partial r, \boldsymbol{e}_{\hat{\theta}}=(1 / r) \partial / \partial \theta$, $\boldsymbol{e}_{\hat{\phi}}=(1 / r \sin \theta) \partial / \partial \phi$ are easily seen to be

$$
\mathcal{E}_{\hat{r} \hat{r}}=-\frac{2 M}{r^{3}}, \quad \mathcal{E}_{\hat{\theta} \hat{\theta}}=\mathcal{E}_{\hat{\phi} \hat{\phi}}=+\frac{M}{r^{3}},
$$

[Eqs. (4.1) above], which are symmetric and trace-free as expected. The field lines associated with this tidal field are easily seen to be those depicted in Fig. 2 above.

For the effects of the spin angular momentum, we shall think of the spinning body as "dragging space into motion" with a velocity and angular velocity (relative to our Cartesian coordinates) given by

$$
\frac{d \boldsymbol{x}_{\text {space }}}{d t} \equiv \boldsymbol{v}_{\text {space }}=-\boldsymbol{\beta}=\frac{2 \boldsymbol{S}}{r^{2}} \times \boldsymbol{n} ;, \quad \frac{d \phi_{\text {space }}}{d t}=\omega=\frac{2 S}{r^{3}}
$$




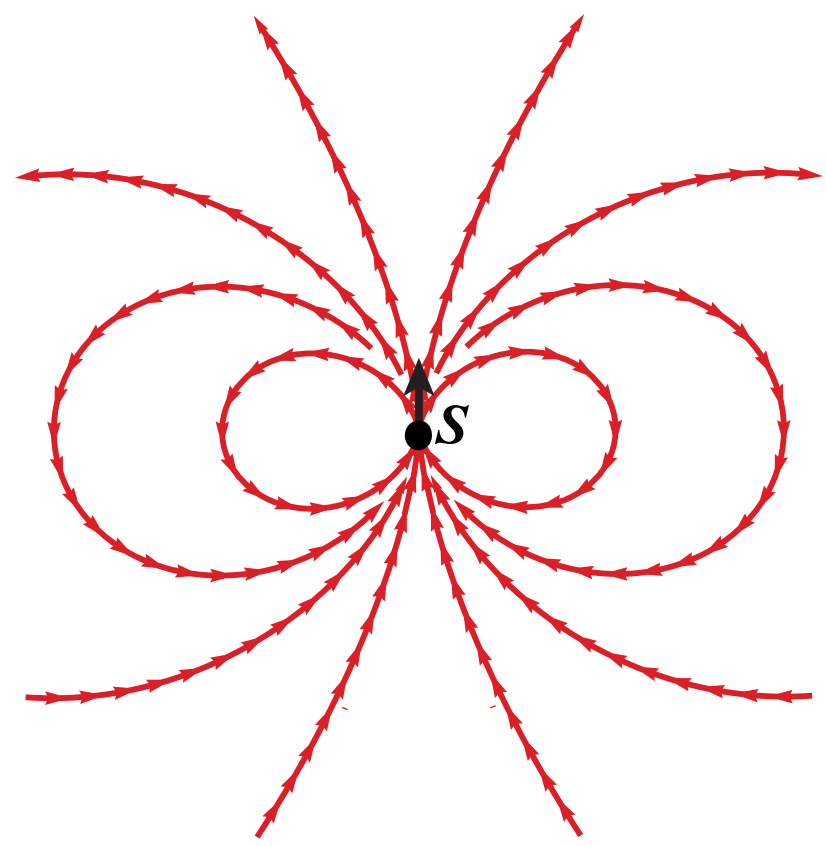

FIG. 4: For a weakly gravitating, slowly rotating body with spin angular momentum $\boldsymbol{S}$ : the dipolar frame-dragging angular velocity relative to inertial frames at infinity, $\boldsymbol{\Omega}_{\mathrm{fd}}$. The arrows are all drawn with the same length rather than proportional to the magnitude of $\boldsymbol{\Omega}_{\mathrm{fd}}$.

[cf. the $\delta_{j k}\left(d x^{j}+\beta^{j} d t\right)\left(d x^{k}+\beta^{k} d t\right)$ term in the metric (5.1a) and the $(d \phi-\omega d t)^{2}$ term in the metric (5.1d)]. Just as the vorticity $\boldsymbol{\nabla} \times \boldsymbol{v}$ of a nonrelativistic fluid with velocity field $\boldsymbol{v}(\boldsymbol{x})$ is twice the angular velocity $\boldsymbol{\Omega}$ of rotation of a fluid element relative to an inertial reference frame, so the vorticity associated with the "space motion", $\boldsymbol{\nabla} \times \boldsymbol{v}_{\text {space, }}$, turns out to be twice the vectorial angular velocity of an inertial-guidance gyroscope relative to inertial reference frames far from the body ("at infinity") - or equivalently, relative to our spatial Cartesian coordinates $\{x, y, z\}$, which are locked to inertial frames at infinity. In formulas: Let $\boldsymbol{\sigma}$ be a unit vector along the spin angular momentum vector of an inertial-guidance gyroscope. Viewed as a vector in our Cartesian basis, it precesses

$$
d \boldsymbol{\sigma} / d t=\boldsymbol{\Omega}_{\mathrm{fd}} \times \boldsymbol{\sigma},
$$

with a frame-dragging vectorial angular velocity equal to half the vorticity of space viewed as a fluid:

$$
\begin{aligned}
\boldsymbol{\Omega}_{\mathrm{fd}} & =\frac{1}{2} \boldsymbol{\nabla} \times \boldsymbol{v}_{\text {space }}=-\frac{1}{2} \nabla \times \boldsymbol{\beta}=-\frac{\mathbf{1}}{\mathbf{2}} \mathbf{H} \\
& =-\left[\frac{\boldsymbol{S}-3(\boldsymbol{S} \cdot \boldsymbol{n}) \boldsymbol{n}}{r^{3}}\right] ;
\end{aligned}
$$

see e.g. Eq. (25.14) of [37], or Eq. (40.37) of [14]. This dipolar frame-dragging angular velocity is shown in Fig. 4.

For dynamical black holes and other strong-gravity, dynamical situations, it is not possible to measure gyro- scopic precession with respect to inertial frames at infinity, since there is no unambiguous way to compare vectors at widely separated events. ${ }^{4}$

On the other hand, we can, in general, measure the precession of inertial-guidance gyroscopes at one event, with respect to inertial frames at a neighboring event i.e., we can measure differential frame dragging as embodied in the frame-drag field (magnetic part of the Weyl tensor) $\mathcal{B}_{i j}$. In our weak-gravity, slow-motion situation, this frame-drag field is equal to the gradient of $\boldsymbol{\Omega}_{\mathrm{fd}}$ (Eq. (5.45b) of [42]):

$$
\mathcal{B}=\nabla \Omega_{\mathrm{fd}}, \quad \text { i.e. } \mathcal{B}_{j k}=\Omega_{\mathrm{fd} j, k} .
$$

For our weakly gravitating, spinning body, $\boldsymbol{\Omega}_{\mathrm{fd}}$ has the dipolar form (5.8), so the frame-drag field is

$$
\mathcal{B}_{j k}=\frac{3}{r^{4}}\left[2 S_{(j} n_{k)}+(\boldsymbol{S} \cdot \boldsymbol{n})\left(\delta_{j k}-5 n_{j} n_{k}\right)\right] .
$$

Here the parentheses on the subscripts indicate symmetrization. In spherical polar coordinates, the components of this frame-drag field are:

$$
\begin{aligned}
& \mathcal{B}_{\hat{r} \hat{r}}=-2 \mathcal{B}_{\hat{\theta} \hat{\theta}}=-2 \mathcal{B}_{\hat{\phi} \hat{\phi}}=\frac{-6 S \cos \theta}{r^{4}}, \\
& \mathcal{B}_{\hat{r} \hat{\theta}}=\mathcal{B}_{\hat{\theta} \hat{r}}=-\frac{3 S \sin \theta}{r^{4}}
\end{aligned}
$$

For this (and any other axially symmetric) frame-drag field, one of the three sets of vortex lines is along the $\phi$ direction (i.e. the $\boldsymbol{S} \times \boldsymbol{x}$ direction), i.e. it is axial; and the other two are poloidal. By computing the eigenvectors of the tensor (5.10) and then drawing the curves to which they are tangent, one can show that the body's vortex lines have the forms shown in Fig. 3 above.

Notice that the poloidal, negative vorticity vortex lines (the poloidal red curves in Fig. 3) all emerge from the north polar region of the spinning body, encircle the body, and return back to the north polar region.

Why do these have negative rather than positive vorticity? Choose the eigendirection $\boldsymbol{p}$ at the body's north pole to point away from the body. The body drags inertial frames in a right-handed manner (counterclockwise as seen looking down on the north pole), and the frame dragging is stronger at the tail of $\boldsymbol{p}$ (nearer the body) than at the tip, so the frame-dragging angular velocity decreases from tail to tip, which means it is more lefthanded (clockwise) at the tip than the tail; it has negative vorticity.

\footnotetext{
4 There is an exception: One can introduce additional geometric structure, e.g, an auxiliary flat spacetime, that provides a way of carrying a reference frame inward from infinity to all other locations and thereby compare vectors at different events. Some of us have used this approach to localize linear momentum in the gravitational field around black holes [40, 41]. However the auxiliary structure has great arbitrariness, and for the vortex and tendex concepts of this paper there is no need for such auxiliary structure, so we eschew it.
} 
The poloidal, positive vorticity vortex lines (the poloidal blue curves in Fig. 3) all emerge from the body's south polar region, swing around the body, and return to the south polar region.

The azimuthal vortex lines have negative vorticity above the hole's equatorial plane (blue azimuthal circles) and positive vorticity below the hole's equatorial plane (red azimuthal circles).

\section{B. Two stationary, weakly gravitating, spinning point particles with opposite spins}

Consider, next, two weakly gravitating, spinning point particles with opposite spins, sitting side-by-side. Place the particles (named $A$ and $B$ ) on the $x$-axis, at locations $\left\{x_{A}, y_{A}, z_{A}\right\}=\{+a, 0,0\},\left\{x_{B}, y_{B}, z_{B}\right\}=\{-a, 0,0\}$ and give them vectorial spins $\boldsymbol{S}_{A}=S \boldsymbol{e}_{z}, \boldsymbol{S}_{B}=-S \boldsymbol{e}_{z}$. Then the frame-drag angular velocity relative to inertial frames at infinity is

$$
\begin{aligned}
\boldsymbol{\Omega}_{\mathrm{fd}}= & -\frac{\boldsymbol{S}_{A}-3\left(\boldsymbol{S}_{A} \cdot \boldsymbol{n}_{A}\right) \boldsymbol{n}_{A}}{r_{A}^{3}} \\
& -\frac{\boldsymbol{S}_{B}-3\left(\boldsymbol{S}_{B} \cdot \boldsymbol{n}_{B}\right) \boldsymbol{n}_{A}}{r_{B}{ }^{3}},
\end{aligned}
$$

where $r_{A}=\left|\boldsymbol{x}-\boldsymbol{x}_{A}\right|$ and $r_{B}=\left|\boldsymbol{x}-\boldsymbol{x}_{B}\right|$ are the distances to the particles and $\boldsymbol{n}_{A}=\left(\boldsymbol{x}-\boldsymbol{x}_{A}\right) / r_{A}$ and $\boldsymbol{n}_{B}=\left(\boldsymbol{x}-\boldsymbol{x}_{B}\right) / r_{B}$ are unit vectors pointing from the particles' locations to the field point; cf. Eq. (5.8). This vector field is plotted in Fig. 5(a). It has just the form one might expect from the one-spin field of Fig. 4.

For these two spinning particles, the frame-drag field (gradient of Eq. (5.12)] is

$$
\begin{aligned}
\mathcal{B}_{j k} & =\frac{3}{r_{A}^{4}}\left[2 S_{A}^{(j} n_{A}^{k)}+\left(\boldsymbol{S}_{A} \cdot \boldsymbol{n}_{A}\right)\left(\delta^{j k}-5 n_{A}^{j} n_{A}^{k}\right)\right] \\
& +\frac{3}{r_{B}^{4}}\left[2 S_{B}^{(j} n_{B}^{k)}+\left(\boldsymbol{S}_{B} \cdot \boldsymbol{n}_{B}\right)\left(\delta^{j k}-5 n_{B}^{j} n_{B}^{k}\right)\right]
\end{aligned}
$$

[cf. Eq. (5.10)], where we have moved the vector and tensor indices up for simplicity of notation. (In our Cartesian basis, there is no difference between up and down indices.)

The best two-dimensional surface on which to visualize vortex lines of this $\mathcal{B}$ is the $x-z$ plane (the plane formed by the particles' spins and their separation vector). The system is reflection symmetric through this plane. On this plane, one of the principal directions of $\mathcal{B}$ is orthogonal to it (in the $y$ direction); the other two lie in the plane and are tangent to the in-plane vortex lines. By computing the eigendirections of $\mathcal{B}$ [i.e., of the tensor (5.13)] and mapping out their tangent vortex lines, and checking the sign of $\mathcal{B}_{p p}$ along their tangent directions $\boldsymbol{p}$, we obtain Fig. 5.

Note that, as for a single spinning particle (Fig. 3), so also here for two spins, the negative-vorticity vortex lines
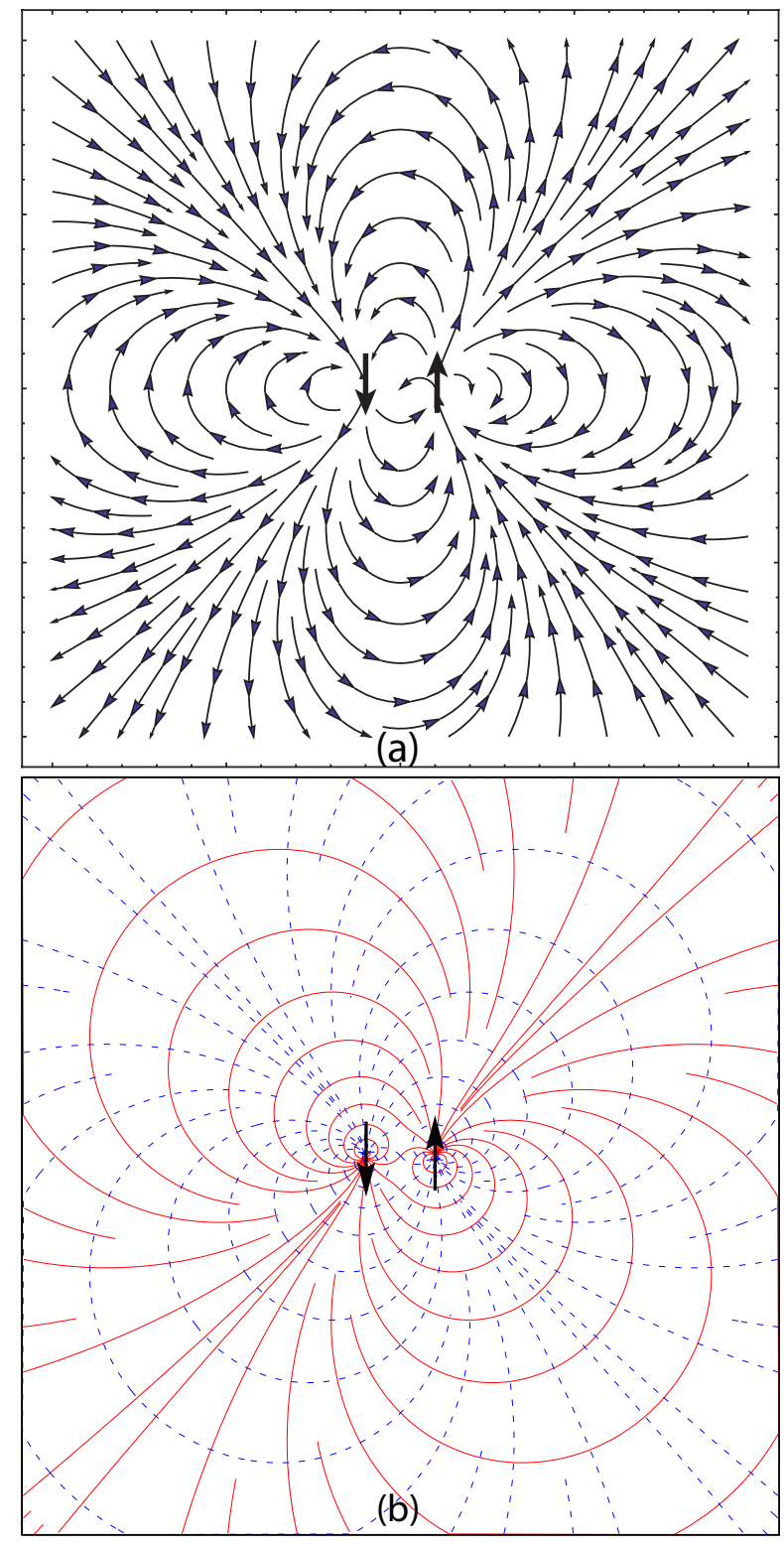

FIG. 5: For two stationary point particles sitting side by side with their spins in opposite directions (thick black arrows): two types of streamlines in the plane of reflection symmetry formed by the particles' spins and their separation vector. Top: The frame-dragging angular velocity $\boldsymbol{\Omega}_{\mathrm{fd}}$ and its streamlines, with the arrows all drawn at the same length rather than proportional to the magnitude of $\boldsymbol{\Omega}_{\mathrm{fd}}$. Bottom: The two sets of vortex lines of the frame-drag field $\mathcal{B}$. The negative-vorticity vortex lines are solid and colored red, and the positive-vorticity ones are dashed and blue. In this figure, as in preceding figures, the colors are not weighted by the lines' vorticities, but only by the signs of the vorticities.

(solid red curves) emerge from the tips of the spins and the positive-vorticity vortex lines (dashed blue curves) emerge from their tails. For a single spin, the negativevorticity vortex lines emerge from the tip, travel around the body, and return to the same tip. Here, the lines 
close to each spinning body leave and enter the same body's tip, but the majority emerge from one body's tip, travel around that body and enter the other body's tip. Similarly the positive-vorticity vortex lines (dashed and blue) emerge from one body's tail, travel around that body, and enter the other body's tail (aside from the lines near each body that exit and return to the same body's tail).

The collection of solid red vortex lines near each arrow tip in Fig. 5(b) constitutes a negative-vorticity framedrag vortex, and the collection of dashed blue vortex lines near each arrow tail is a positive-vorticity vortex.

\section{The two spinning particles viewed from afar: Stationary, quadrupolar frame-drag field}

When viewed from afar, the two spinning bodies produce a current-quadrupole gravitational field with quadrupole moment (e.g. Eq. (5.28b) of [36])

$$
\begin{aligned}
\mathcal{S}_{p q} & =\left(\int j_{p} x_{q} d^{3} x\right)^{\mathrm{STF}}=\left(S_{p} a_{q}+\left(-S_{p}\right)\left(-a_{q}\right)\right)^{\mathrm{STF}} \\
& =S_{p} a_{q}+S_{q} a_{p}-\frac{2}{3}(\boldsymbol{S} \cdot \boldsymbol{a}) \delta_{p q}
\end{aligned}
$$

Here $j_{p}=S_{p} \delta(\boldsymbol{x}-\boldsymbol{a})-S_{p} \delta(\boldsymbol{x}+\boldsymbol{a})$ is the angular momentum density. Since the only nonzero components of $\boldsymbol{S}$ and $\boldsymbol{a}$ are $S_{z}=S$ and $a_{x}=a$, the only nonzero components of the current quadrupole moment are

$$
\mathcal{S}_{x z}=\mathcal{S}_{z x}=S a .
$$

The frame-drag-induced velocity of space (negative of lapse function) for this current quadrupole, and the frame-drag angular velocity and frame-drag tensor field are

$$
\begin{aligned}
\boldsymbol{v}_{\text {space }} & =-\boldsymbol{\beta}=\frac{4 \boldsymbol{n} \times \mathcal{S} \cdot \boldsymbol{n}}{r^{3}}, \\
\boldsymbol{\Omega}_{\mathrm{fd}} & =\frac{1}{2} \boldsymbol{\nabla} \times \boldsymbol{v}_{\text {space }}, \quad \mathcal{B}=\nabla \boldsymbol{\Omega}_{\mathrm{fd}} .
\end{aligned}
$$

[e.g. Eq. (10.6b) of [36]; also Eqs. (5.8) and (5.9) above]. Inserting Eq. (5.15) for the quadrupole moment into Eqs. (5.16), and plotting $\boldsymbol{\Omega}_{\mathrm{fd}}$ and the vortex lines of $\mathcal{B}$ in the $x-z$ plane, we obtain the graphs shown in Fig. 6 .

Notice that the current-quadrupolar frame-drag angular velocity in Fig. 6a is, indeed, the same as that for two oppositely directed spins (Fig. 5a) in the limit that the spins' separation goes to zero - i.e., as seen from afar; and the current-quadrupolar vortex lines of the framedrag tensor field (Fig. 6b) is the vanishing-separation limit of that for the two oppositely directed spins (Fig. $5 \mathrm{~b})$.

Here, as for finitely separated spinning particles, there are two red frame-drag vortexes, one emerging from the origin in the upper right direction, the other in the lowerleft direction; and similarly, there are two blue framedrag vortexes, one emerging in the upper left direction and the other in the lower right direction.
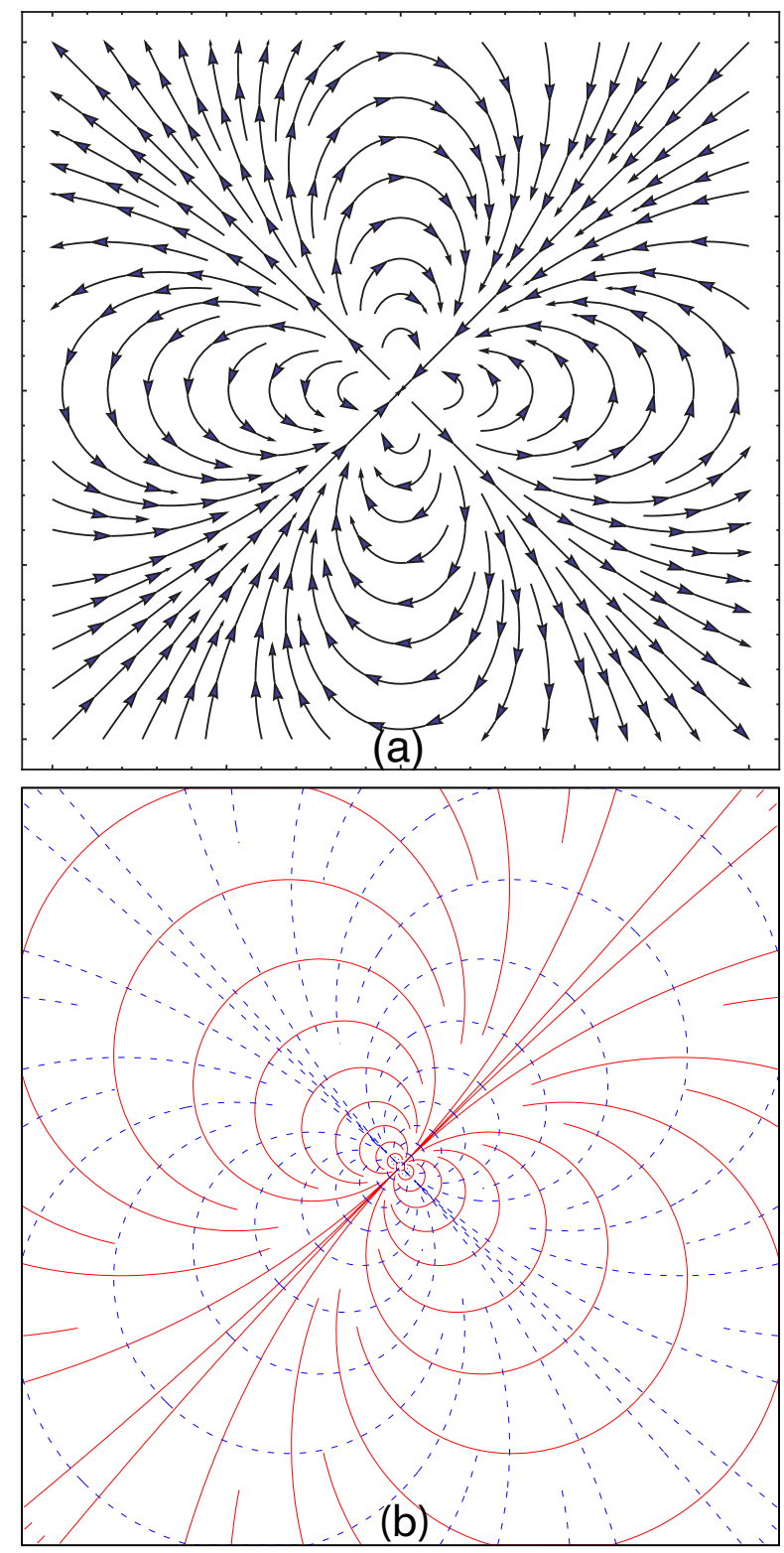

FIG. 6: Current-quadrupolar streamlines associated with the two stationary spinning particles of Fig. 5, for which the current quadrupole moment has nonzero components $\mathcal{S}_{x z}=$ $\mathcal{S}_{z x}=S a$. (a) The frame-dragging angular velocity $\boldsymbol{\Omega}_{\mathrm{fd}}$ and its streamlines, and (b) the two sets of vortex lines, in the $x-z$ plane. Figure (b) also describes the tendex lines for a static mass-quadrupolar particle whose only nonzero quadrupolemoment components are $\mathcal{I}_{x z}=\mathcal{I}_{z x}$.

\section{Static, quadrupolar tidal field and its tendex lines and tendexes}

For an idealized static particle with time independent mass quadrupole moment $\mathcal{I}_{p q}$ and all other moments (including the mass) vanishing, the squared lapse function is $\alpha^{2}=1+2 \Phi=-\left(\mathcal{I}_{p q} / r\right)_{, p q}[36]$, where $\Phi$ is the Newtonian gravitational potential. Therefore, the particle's 
tidal field $\mathcal{E}_{j k}=\Phi_{, j k}[\mathrm{Eq} .(5.8)]$ is

$$
\mathcal{E}_{j k}=-\frac{1}{2}\left(\frac{\mathcal{I}_{p q}}{r}\right)_{, p q j k} .
$$

For comparison, for a particle with time-independent current quadrupole moment $\mathcal{S}_{p q}$, the shift function is $\beta_{j}=(-4 / 3) \epsilon_{j p q}\left(\mathcal{S}_{p k} / r\right)_{, k q}$, which implies that the framedrag field is [Eqs. (5.16)]

$$
\mathcal{B}_{j k}=-\frac{2}{3}\left(\frac{\mathcal{S}_{p q}}{r}\right)_{, p q j k} .
$$

Notice that, once the differing normalization conventions (2.18) are accounted for, Eqs. (5.17) and (5.18) are the same, as required by the duality relations (2.14) and (2.18). This means that, for a static current quadrupole whose only nonzero components are $\mathcal{I}_{x z}=\mathcal{I}_{z x}$, the tendex lines will have precisely the same forms as the vortex lines of the static current quadrupole (5.15); i.e., they will have the forms shown in Fig. 6b. In this case there are two negative-tendicity (solid red) tidal tendexes, one emerging from the origin in the upper right direction, and the other in the lower-left direction; and there are two positive-tendicity (dashed blue) tidal tendexes, one emerging in the upper left direction and the other in the lower right direction.

\section{GRAVITATIONAL WAVES AND THEIR GENERATION}

We turn now to dynamical situations, which we describe using linearized gravity. We first discuss the tendex and vortex structure of plane gravitational waves. We then examine wave generation by time varying multipolar fields, and the accompanying tendex and vortex structures of these systems.

\section{A. Plane gravitational wave}

In this section, we will describe the features of $\mathcal{E}$ and $\mathcal{B}$ for plane gravitational waves, and connect our observations to the linearized-gravity and Newman-Penrose (NP) formalisms. In Appendix A we review the NewmanPenrose formalism and its connection to the spatial tensors $\mathcal{E}$ and $\mathcal{B}$.

Consider gravitational wave propagation in an asymptotically flat spacetime, in transverse-traceless (TT) gauge. Near future null infinity, $\mathcal{I}^{+}$, we can linearize around a Minkowski background and obtain

$$
\mathcal{E}_{i j}=-\frac{1}{2} \partial_{0}^{2} h_{i j}, \quad \mathcal{B}_{i j}=-\frac{1}{2} \epsilon_{i}{ }^{p q} n_{p} \partial_{0}^{2} h_{q j} .
$$

It is convenient to expand these expressions in terms of the two gravitational wave polarization tensors, $e_{i j}^{+}$and $e_{i j}^{\times}$

$$
\begin{aligned}
\mathcal{E}_{i j} & =-\frac{1}{2}\left(\ddot{h}_{+} e_{i j}^{+}+\ddot{h}_{\times} e_{i j}^{\times}\right), \\
\mathcal{B}_{i j} & =-\frac{1}{2}\left(\ddot{h}_{+} e_{i j}^{\times}-\ddot{h}_{\times} e_{i j}^{+}\right),
\end{aligned}
$$

where $e_{i j}^{+}$and $e_{i j}^{\times}$are symmetric, trace-free, and orthogonal to the waves' propagation direction. Letting the unit-norm vector $\mathbf{e}_{\hat{1}}$ denote the direction of propagation of the gravitational wave, then one can expand the polarization tensors in terms of the remaining two vectors of an orthonormal triad, $\mathbf{e}_{\hat{2}}$ and $\mathbf{e}_{\hat{3}}$, as

$$
\begin{aligned}
& e_{+}=e_{\hat{2}} \otimes e_{\hat{2}}-e_{\hat{3}} \otimes e_{\hat{3}} \\
& e_{\times}=e_{\hat{2}} \otimes e_{\hat{3}}+e_{\hat{3}} \otimes e_{\hat{2}}
\end{aligned}
$$

Consider first $\mathrm{a}+$ polarized wave. We have that

$$
\mathcal{E}=-\frac{1}{2} \ddot{h}_{+} \boldsymbol{e}^{+}=\frac{1}{2}\left[\left(-\ddot{h}_{+}\right) \boldsymbol{e}_{\hat{2}} \otimes \boldsymbol{e}_{\hat{2}}+\ddot{h}_{+} \boldsymbol{e}_{\hat{3}} \otimes \boldsymbol{e}_{\hat{3}}\right]
$$

so we see that $\mp \ddot{h}_{+} / 2$ are the two eigenvalues of $\mathcal{E}$ (the two tendicities), and $\boldsymbol{e}_{\hat{2}}$ and $\boldsymbol{e}_{\hat{3}}$ are the two corresponding eigenvectors. Now, define a second basis locally rotated at each point by $\pi / 4=45^{\circ}$,

$$
\left(\begin{array}{c}
\tilde{\boldsymbol{e}}_{\hat{2}} \\
\tilde{\boldsymbol{e}}_{\hat{3}}
\end{array}\right)=\left(\begin{array}{cc}
\cos \frac{\pi}{4} & \sin \frac{\pi}{4} \\
-\sin \frac{\pi}{4} & \cos \frac{\pi}{4}
\end{array}\right)\left(\begin{array}{c}
\boldsymbol{e}_{\hat{2}} \\
\boldsymbol{e}_{\hat{3}}
\end{array}\right) .
$$

Then, a simple calculation shows that

$$
\boldsymbol{e}_{\times}=\tilde{\boldsymbol{e}}_{\hat{2}} \otimes \tilde{\boldsymbol{e}}_{\hat{2}}-\tilde{\boldsymbol{e}}_{\hat{3}} \otimes \tilde{\boldsymbol{e}}_{\hat{3}},
$$

and one can immediately see that $\mathcal{B}$ is diagonal in this new basis

$$
\mathcal{B}=-\frac{1}{2} \ddot{h}_{+} \boldsymbol{e}^{\times}=-\frac{1}{2}\left[\ddot{h}_{+} \tilde{\boldsymbol{e}}_{\hat{2}} \otimes \tilde{\boldsymbol{e}}_{\hat{2}}-\ddot{h}_{+} \tilde{\boldsymbol{e}}_{\hat{3}} \otimes \tilde{\boldsymbol{e}}_{\hat{2}}\right]
$$

The eigenvalues of $\mathcal{B}$ (the vorticities), like those of $\mathcal{E}$ (the tendicities), are $\mp \ddot{h}_{+} / 2$, but $\mathcal{B}$ 's eigenvectors, $\tilde{\boldsymbol{e}}_{\hat{2}}$ and $\tilde{\boldsymbol{e}}_{\hat{3}}$, are locally rotated by $\pi / 4$ compared to those of $\mathcal{E}$. Correspondingly, the vortex lines of $h_{+}$must be locally rotated by $\pi / 4$ with respect to the tendex lines.

The local rotation of the tendex and vortex lines is most transparent for a plane gravitational wave. In Fig. 7, we show the tendex and vortex lines of a plane gravitational wave propagating out of the page (i.e. $\mathbf{e}_{\hat{1}}=\mathbf{e}_{\hat{z}}$ is the propagation direction). Because the eigenvectors of $\mathcal{E}$ are $\mathbf{e}_{\hat{2}}=\mathbf{e}_{\hat{x}}$ and $\mathbf{e}_{\hat{3}}=\mathbf{e}_{\hat{y}}$, the tendex lines are the lines of constant $x$ and $y$, illustrated by red (solid) lines and blue (dashed) lines, respectively, on the left of Fig. 7. Similarly, the vortex lines are lines of constant $x \pm y$, again drawn as blue (dashed) lines and red (solid) lines, respectively. The tendicity (vorticity) has constant magnitude along the lines, but the two sets of tendex (vortex) lines have opposite sign; consequently, the tidal (frame-drag) field produces a stretching (counterclockwise differential precession) along the solid red direction and a squeezing 


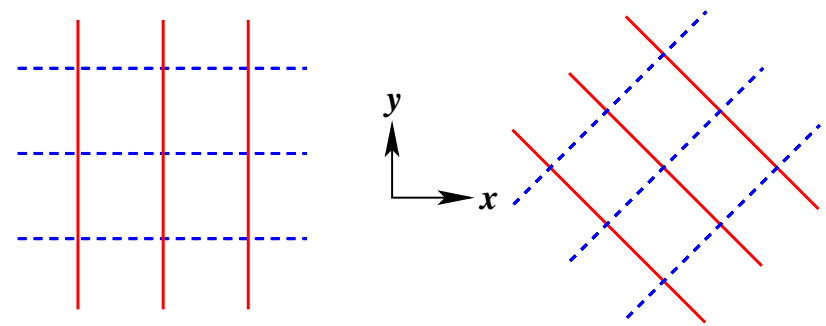

FIG. 7: The tendex lines (left) and vortex lines (right) of a plane gravitational wave propagating in the $z$-direction (out of the picture). The tendex lines are lines of constant $x$ and $y$, and the vortex lines are rotated by $\pi / 4$ (lines of constant $x \pm y$ ). The blue (dashed) curves correspond to positive tendicity and vorticity (squeezing and clockwise differential precessing, respectively) and the red (solid) curves denote negative tendicity and vorticity (stretching and counterclockwise precessing). The tendicity (vorticity) is constant along a tendex line (vortex line), and the tendicity (vorticity) of a red line is equal in magnitude but opposite in sign to that of a blue (dashed) line.

(clockwise differential precession) of the same magnitude along the dashed blue direction.

More generally, gravitational waves will contain both + and $\times$ polarizations, and to study their vortex and tendex lines, it will be useful to express the electric and magnetic tensors in the spatial orthonormal basis $\left(\boldsymbol{e}_{\hat{1}}, \boldsymbol{e}_{\hat{2}}, \boldsymbol{e}_{\hat{3}}\right)$. They can be written conveniently as matrices:

$$
\begin{aligned}
& \mathcal{E}_{\hat{a} \hat{b}}=\frac{1}{2}\left(\begin{array}{c|cc}
0 & 0 & 0 \\
\hline 0 & -\ddot{h}_{+} & -\ddot{h}_{\times} \\
0 & -\ddot{h}_{\times} & \ddot{h}_{+}
\end{array}\right), \\
& \mathcal{B}_{\hat{a} \hat{b}}=\frac{1}{2}\left(\begin{array}{c|cc}
0 & 0 & 0 \\
\hline 0 & \ddot{h}_{\times} & -\ddot{h}_{+} \\
0 & -\ddot{h}_{+} & -\ddot{h}_{\times}
\end{array}\right) .
\end{aligned}
$$

It is useful to introduce an associated Newman-Penrose null tetrad consisting of two real null vectors, $\vec{l}$ (along the waves' propagation direction) and $\vec{n}$, and a conjugate pair of complex null vectors $\vec{m}$ and $\vec{m}^{*}$ given by

$$
\begin{gathered}
\vec{l}=\frac{1}{\sqrt{2}}\left(\vec{e}_{\hat{0}}+\vec{e}_{\hat{1}}\right), \quad \vec{n}=\frac{1}{\sqrt{2}}\left(\vec{e}_{\hat{0}}-\vec{e}_{\hat{1}}\right), \\
\vec{m}=\frac{1}{\sqrt{2}}\left(\vec{e}_{\hat{2}}+i \vec{e}_{\hat{3}}\right), \quad \vec{m}^{*}=\frac{1}{\sqrt{2}}\left(\vec{e}_{\hat{2}}-i \vec{e}_{\hat{3}}\right)
\end{gathered}
$$

[Eqs. (A1) of Appendix A]. For plane waves on a Minkowski background, the NP curvature scalar that characterizes the radiation is

$$
\Psi_{4}=C_{\mu \nu \rho \sigma} n^{\mu} m^{* \nu} n^{\rho} m^{* \sigma}=-\ddot{h}_{+}+i \ddot{h}_{\times},
$$

so we can compactly rewrite Eqs. (6.9) as

$$
\mathcal{E}_{\hat{a} \hat{b}}+i \mathcal{B}_{\hat{a} \hat{b}}=\frac{1}{2}\left(\begin{array}{c|cc}
0 & 0 & 0 \\
\hline 0 & \Psi_{4} & i \Psi_{4} \\
0 & i \Psi_{4} & -\Psi_{4}
\end{array}\right) .
$$

This expression holds for any plane gravitational wave propagating in the $\vec{e}_{\hat{1}}$ direction.

For any outgoing gravitational wave in an asymptotically flat space, as one approaches asymptotic null infinity the general expression (A3) for $\mathcal{E}_{\hat{a} \hat{b}}+i \mathcal{B}_{\hat{a} \hat{b}}$ reduces to expression (6.12), because all the curvature scalars except $\Psi_{4}$ vanish due to the peeling property of the Weyl scalars near null infinity. Further discussion of the tidal and frame-drag fields of radiation near null infinity and their tendex and vortex lines is given in [28].

It is helpful to draw some simple analogies between gravitational and electromagnetic plane waves. For a generic mixture of + and $\times$ polarizations, the magnitudes of the nonvanishing eigenvalues of both $\mathcal{E}$ and $\mathcal{B}$ are simply

$$
\frac{1}{2} \sqrt{\ddot{h}_{+}^{2}+\ddot{h}_{\times}^{2}}=\frac{1}{2}\left|\Psi_{4}\right| .
$$

This mirrors plane waves in electromagnetism, where $|\vec{E}|=|\vec{B}|$ is equal to the sum in quadrature of the magnitudes of the two polarizations. The absent longitudinal components in an electromagnetic plane wave correspond to the vanishing of the eigenvalues for the eigenvectors of $\mathcal{E}$ and $\mathcal{B}$ along the propagation direction. The orthogonality of the vectorial electromagnetic field strengths $\vec{E} \perp \vec{B}$ becomes the $\pi / 4$ rotation between the meshes (Fig. 7) formed by the two transverse eigenvectors of the tensorial quantities $\mathcal{E}$ and $\mathcal{B}$.

\section{B. Gravitational waves from a head-on collision of two black holes}

As an example of the usefulness of this approach, we calculate the tendex and vortex lines at large radii for gravitational waves emitted by the head-on collision of two equal-mass nonspinning black holes. If the holes move along the $z$-axis and we use as our spatial triad the unit vectors of spherical polar coordinates, $\left(\mathbf{e}_{\hat{1}}, \mathbf{e}_{\hat{2}}, \mathbf{e}_{\hat{3}}\right)=$ $\left(\mathbf{e}_{\hat{r}}, \mathbf{e}_{\hat{\theta}}, \mathbf{e}_{\hat{\phi}}\right)=\left(\partial_{r}, r^{-1} \partial_{\theta},(r \sin \theta)^{-1} \partial_{\phi}\right)$, and choose our null tetrad in the usual way (6.10), then we can apply the results described by Fiske et al. [43]; namely that $\Re\left[\Psi_{4}\right]$ is axisymmetric (and, when decomposed into spin-weighted spherical harmonics, is dominated by the $l=2, m=0$ harmonic, $\left.{ }_{-2} Y_{2,0}(\theta, \phi)\right)$; and that $\Im\left[\Psi_{4}\right]=0$. Then the electric and magnetic parts of the Weyl tensor are given by

$$
\begin{aligned}
& \mathcal{E}_{\hat{a} \hat{b}}=\frac{1}{2}\left(\begin{array}{c|cc}
0 & 0 & 0 \\
\hline 0 & \Re\left(\Psi_{4}\right) & 0 \\
0 & 0 & -\Re\left(\Psi_{4}\right)
\end{array}\right), \\
& \mathcal{B}_{\hat{a} \hat{b}}=\frac{1}{2}\left(\begin{array}{c|cc}
0 & 0 & 0 \\
\hline 0 & 0 & \Re\left(\Psi_{4}\right) \\
0 & \Re\left(\Psi_{4}\right) & 0
\end{array}\right),
\end{aligned}
$$

and the eigenvalues of both $\mathcal{E}$ and $\mathcal{B}$ are $\pm \Re\left(\Psi_{4}\right) / 2$. The eigenvectors of $\mathcal{E}$ are the unit vectors $\mathbf{e}_{\hat{\theta}}$ and $\mathbf{e}_{\hat{\phi}}$, and 


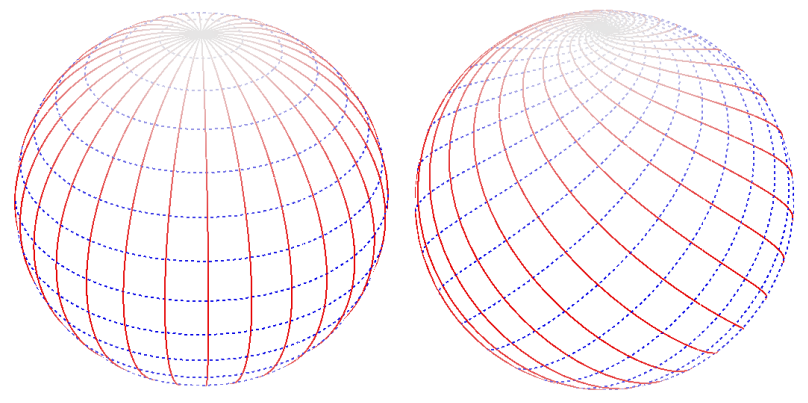

FIG. 8: Tendex lines (left) and vortex lines (right) for the gravitational waves that would arise from the merger of equalmass black holes falling together along the z-axis. The positive tendicity and vorticity lines are shown in blue (dashed) and the negative lines are depicted in red (solid). Each line's intensity is proportional to its tendicity (or vorticity), which varies over the sphere as the dominant spin-weighted spherical harmonic, ${ }_{-2} Y_{2,0}(\theta, \phi) \propto \sin ^{2} \theta$. Dark red and blue near the equator correspond to large-magnitude tendicity and vorticity, and light nearly white colors at the poles indicate that the tendicity and vorticity are small there.

those of $\mathcal{B}$ are $\mathbf{e}_{\hat{\theta}} \pm \mathbf{e}_{\hat{\phi}}$. Thus, the radiation is purely + polarized in this basis. The tendex lines are the lines of constant $\theta$ and $\phi$ on a sphere, and the vortex lines are rotated relative to the tendex lines by $\pi / 4=45^{\circ}$.

We show these lines in Fig. 8; the tendex lines on the left, and the vortex lines on the right. As in Fig. 7, the red (solid) lines correspond to negative tendicity and vorticity, and the blue (dashed) lines denote positive values. The intensity of each line is proportional to the magnitude of its tendicity (or vorticity), which varies over the sphere as ${ }_{-2} Y_{2,0}(\theta, \phi) \propto \sin ^{2} \theta$ (the dominant spherical harmonic). Correspondingly, the dark blue and red regions near the equator represent strong tendicity and vorticity, whereas the light off-white colors near the poles indicate that the tendicity and vorticity are small there.

We remark in passing that the duality of $\mathcal{E}$ and $\mathcal{B}$ implies that, if there were a source of gravitational waves which had a $\Psi_{4}$ that is purely imaginary and equal to the $i \Re\left[\Psi_{4}\right]$ for our colliding black holes, then those waves' vortex lines would be the same as the tendex lines of Fig. 8, and the tendex lines would be the same as the vortex lines of the same figure (but with the sign of the lines' vorticity flipped). One can see this because (i) Eq. (6.11) shows we would have a pure $\times$ polarized wave, and (ii) when we apply the rotation of basis (6.6) to (6.12) under the condition of $\Re\left(\Psi_{4}\right)=0$ we get once again the matrices $(6.14)$, but with $\left(\tilde{\boldsymbol{e}}_{\hat{2}}, \tilde{\boldsymbol{e}}_{\hat{3}}\right)$ as basis vectors and with all instances of $\Re\left(\Psi_{4}\right)$ replaced by $\Im\left(\Psi_{4}\right)$. This duality does not address, however, how to construct a source with a purely imaginary $\Psi_{4}$.

\section{Wave generation by a time varying current quadrupole}

A dynamical current quadrupole moment $\mathcal{S}_{p q}(t)$ generates a metric perturbation described by the $\mathcal{S}_{p q}(t-r) / r$ terms in Eqs. (8.13) of [36]. It is straightforward to show that the corresponding frame-drag field is

$$
\begin{aligned}
\mathcal{B}_{i j}= & \frac{2}{3}\left[-\left(\frac{\mathcal{S}_{p q}}{r}\right)_{, p q i j}+\epsilon_{i p q}\left(\frac{{ }^{(2)} \mathcal{S}_{p m}}{r}\right)_{, q n} \epsilon_{j m n}\right. \\
& \left.+2\left(\frac{(2) \mathcal{S}_{p(i}}{r}\right)_{, j) p}-\left(\frac{{ }^{(4)} \mathcal{S}_{i j}}{r}\right)\right]
\end{aligned}
$$

Here $\mathcal{S}_{p q}$ is to be regarded as a function of retarded time, $t-r$, and the prefixes ${ }^{(2)}$ and ${ }^{(4)}$ mean two time derivatives and four time derivatives. This equation shows explicitly how $\mathcal{B}_{i j}$ in the near zone transitions into $\mathcal{B}_{i j}$ in the wave zone - or equivalently, how rotating (or otherwise time-changing) frame-drag vortexes in the near zone generate gravitational waves.

This transition from near zone to far zone can also be described by the linear approximation to the Maxwelllike equations for the frame-drag field $\mathcal{B}$ and the tidal field $\mathcal{E}$, Eqs. (2.15). These equations govern the manner by which the current-quadrupole near-zone framedrag field (5.18) acquires an accompanying tidal field as it reaches outward into and through the transition zone, to the wave zone. That accompanying tidal field is most easily deduced from the $\mathcal{S}_{p q}(t-r) / r$ terms in the metric perturbation, Eqs. (8.13) of [36]. The result is:

$$
\mathcal{E}_{i j}=\frac{4}{3} \epsilon_{p q(i}\left[-\left(\frac{{ }^{(1)} \mathcal{S}_{p k}}{r}\right)_{, j) k q}+\left(\frac{{ }^{(3)} \mathcal{S}_{j) p}}{r}\right)_{, q}\right] .
$$

In the near zone, the current quadrupole's tidal field [first term of (6.16)] behaves differently from its framedrag field [first term of (6.15)]: it has one additional time derivative and one fewer space derivative. As a result, the tidal field is smaller than the frame-drag field in the near zone by a factor of $r / \lambda$, where $\lambda$ is the reduced wavelength of the emitted gravitational waves.

As one moves outward through the near zone to the transition zone, where $r \sim \lambda$, the tidal field increases in magnitude to become the same strength as the framedrag field. The frame-drag and tidal fields behave this way, because it is the near-zone vortexes that generate the gravitational waves, as discussed above.

In the wave zone, the general current-quadrupole (outgoing-wave) frame-drag field (6.15) reduces to

$$
\mathcal{B}_{\hat{a} \hat{b}}=\frac{4}{3 r}\left[{ }^{(4)} S_{\hat{a} \hat{b}}(t-r)\right]^{\mathrm{TT}} .
$$

Here the indices are confined to transverse directions (the surface of a sphere of constant $r$ ) in the orthonormal basis $\boldsymbol{e}_{\hat{\theta}}, \boldsymbol{e}_{\hat{\phi}}$, and "TT" means "take the transverse, trace- 
less part". From the third of the Maxwell-like equations (2.15), or equally well from the general currentquadrupole tidal field, Eq. (6.16), we infer the wave-zone tidal field:

$$
\mathcal{E}_{\hat{a} \hat{b}}=\frac{4}{3 r}\left[\epsilon_{\hat{c}(\hat{a}}^{(4)} \mathcal{S}_{\hat{b}) \hat{c}}(t-r)\right]^{\mathrm{TT}}
$$

where $\epsilon_{\hat{c} \hat{d}}$ is the 2-dimensional Levi-Civita tensor on the sphere. Since $\mathcal{E}_{\hat{a} \hat{b}}=\mathcal{R}_{\hat{a} \hat{0} \hat{b} \hat{0}}=-\frac{1}{2}^{(2)} h_{\hat{a} \hat{b}}^{\mathrm{TT}}$, where $h_{\hat{a} \hat{b}}^{\mathrm{TT}}$ is the transverse, traceless gravitational-wave field, our wavezone tidal distortion (6.18) agrees with the standard result for the wave-zone current-quadrupole gravitationalwave field (Eq. (4.8) of [36]).

\section{Rotating current quadrupole}

In this section, we will discuss the vortex and tendex lines of a rotating current quadrupole.

A large rotating current quadrupole moment arises during the merger and ringdown of the extreme-kick configuration of a binary black hole (a quasi-circular binary made of identical black holes, whose spins are antialigned and lie in the orbital plane). During the merger, the four vortexes associated with the initial holes' spins get deposited onto the merged horizon's equator, and they then rotate around the final Kerr hole's spin axis at the same rate as their separation vector rotates, generating a large, rotating current quadrupole moment (paper III in this series).

As a simple linearized-gravity model of this late time behavior, imagine that at an initial time $t=0$, the two vortex-generating spins, of magnitude $S$, are separated by a distance $a$ along the $x$ axis and are pointing in the $\pm y$ direction - i.e. they have the same configuration as the static current quadrupole discussed in Sec. $\mathrm{VC}$ above. Then at $t=0$, the spins' current quadrupole moment has as its nonzero components $\mathcal{S}_{x y}=\mathcal{S}_{y x}=S a$ [Eq. (5.15) with the spin axes changed from $z$ to $y$ ]. As time passes, the spins' separation vector and the spins' directions rotate at the same angular velocity $\omega$ so the configuration rotates rigidly. Then it is not hard to show that the current quadrupole moment evolves as

$$
\begin{aligned}
& \mathcal{S}_{x y}=\mathcal{S}_{y x}=S a \cos (2 \omega t), \\
& \mathcal{S}_{x x}=-\mathcal{S}_{y y}=-S a \sin (2 \omega t) .
\end{aligned}
$$

It is straightforward to calculate the frame drag field produced by this quadrupole moment using Eq. (6.15), and to then compute the vortex lines and their vorticities.

The explicit expressions for these lines are somewhat lengthy, and not particularly instructive; but the shapes of the vortex lines and the values of their vorticities are quite interesting.

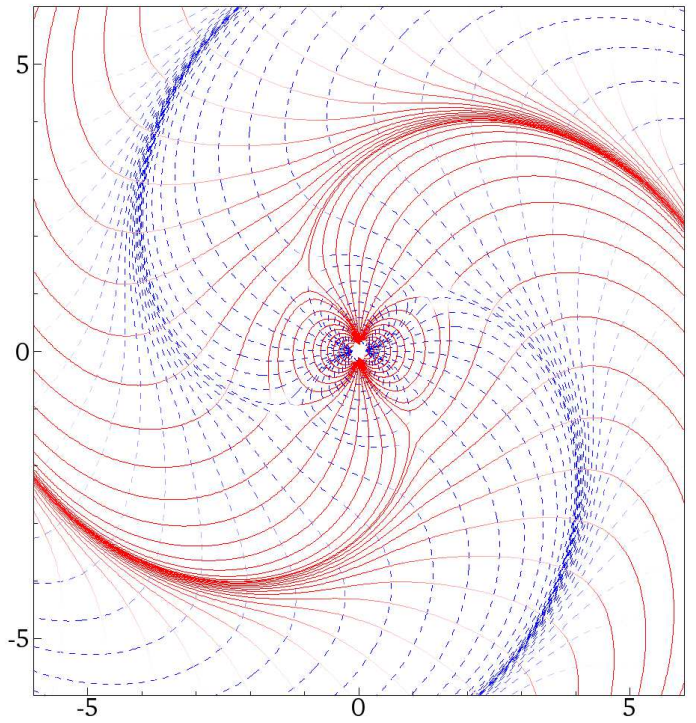

FIG. 9: For a rotating current quadrupole in linearized theory: two families of vortex lines in the plane of reflection symmetry (the $x-y$ plane). The red (solid) curves are lines with negative vorticity, and the blue (dashed) curves are lines of positive vorticity. The color intensity of the curves represents the strength of the vorticity, but rescaled by $(k r)^{5} /\left[1+(k r)^{4}\right]$ (with $k$ the wave number) to remove the vorticity's radial decay. We see the quadrupolar near zone pattern and the transition into the induction zone. In the induction zone, the pattern carries four "triradius" singular points [44] in each family of curves, necessitated for the transition from the static quadrupole pattern to the spiraling radiation pattern. This same figure also describes the tendex lines of a rotating mass quadrupole (see the end of Sec. VIF).

\section{Vortex and tendex lines in the plane of reflection symmetry}

There are two sets of vortex lines that lie in the $x$ $y$ plane (the plane of reflection symmetry) and one set that passes orthogonally through this plane. We show the in-plane vortex lines in Figs. 9 and 10. The two figures depict the negative-vorticity vortex lines by red (solid) curves and the positive-vorticity lines by blue (dashed) curves. The darkness of the lines is proportional to the vorticity; dark red (blue) indicates strong negative (positive) vorticity, and light red (blue) indicates weaker vorticity. To remove the effects of the radial dependence in the coloring, we have scaled the vorticity by $(k r)^{5} /\left[1+(k r)^{4}\right]$, where $k=1 / \lambda=2 \omega$ is the wave number of the radiation. Figure 9 shows the region of the near zone that is difficult to see in Fig. 10, an equivalent figure that spans a larger region of the $x-y$ plane. As one can see from the figures, the two sets of lines have the same pattern, but are rotated with respect to each other by $\pi / 2=90^{\circ}$.

In the near zone (inner region of Fig. 9), the vortex-line pattern is the same as for the static current quadrupole 


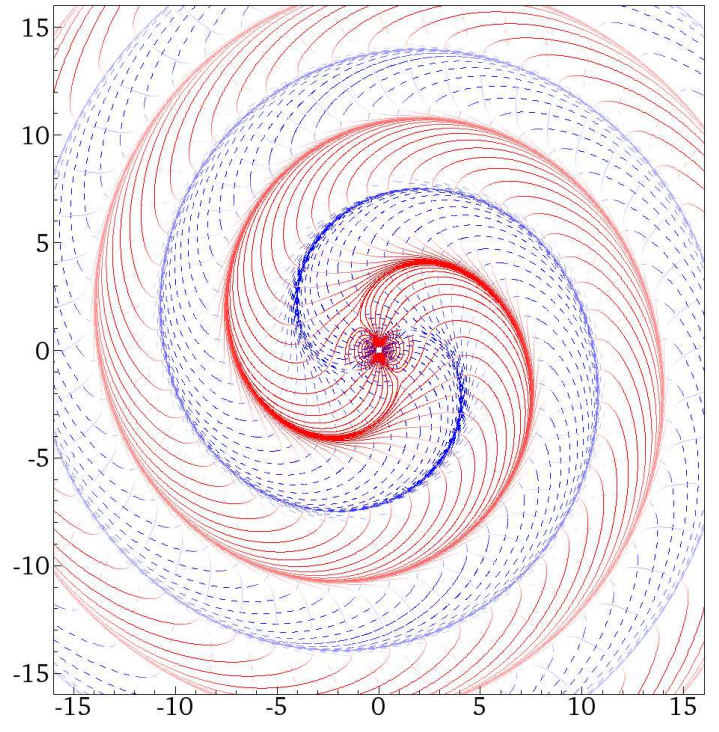

FIG. 10: Same as Fig. 9 but zoomed out to show the wave zone. In the wave zone, the lines generically collect into spirals, which form the boundaries of vortexes (regions of concentrated vorticity).

of Fig. 6b. At the transition to the wave zone, the vortex lines fail to curve back into the central region and instead bend outward, joining a wave-zone spiral pattern.

That spiral pattern consists of four vortexes (regions of concentrated vorticity) that spiral outward and backward as the quadrupole rotates. These four regions of alternating positive and negative vorticity are bounded by tight clusters of vortex lines, just outside of which the sign of the dominant vorticity changes.

This same rotating vortex structure occurs in the case of an $l=2, m=2$, odd-parity (current quadrupolar) perturbation of a Schwarzschild black hole (Paper II in this series). There the horizon vorticity pattern takes the place of the current quadrupole.

In Fig. 11 we indicate the structure of the tendex lines on the equatorial plane. Because the symmetry properties of the system imply different constraints on the tendex field than on the vortex field, some explanation is needed. The plane in which this and the previous two figures are drawn is a plane of reflection symmetry for the problem. However, because the source is a pure current quadrupole, it must be antisymmetric under reflection across this plane (as such a reflection is a parity inversion). The vorticity, which itself has an odd parity relationship with its source, is symmetric under this reflection, constraining the vortex lines to be either tangent or orthogonal to the plane, as noted above. The tendicity is antisymmetric under this reflection, so one family of lines can be tangent to the plane, so long as it has zero tendicity, and two other families of lines must cross the plane at equal and opposite inclinations, with equal and opposite tendicities, such that they are exchanged

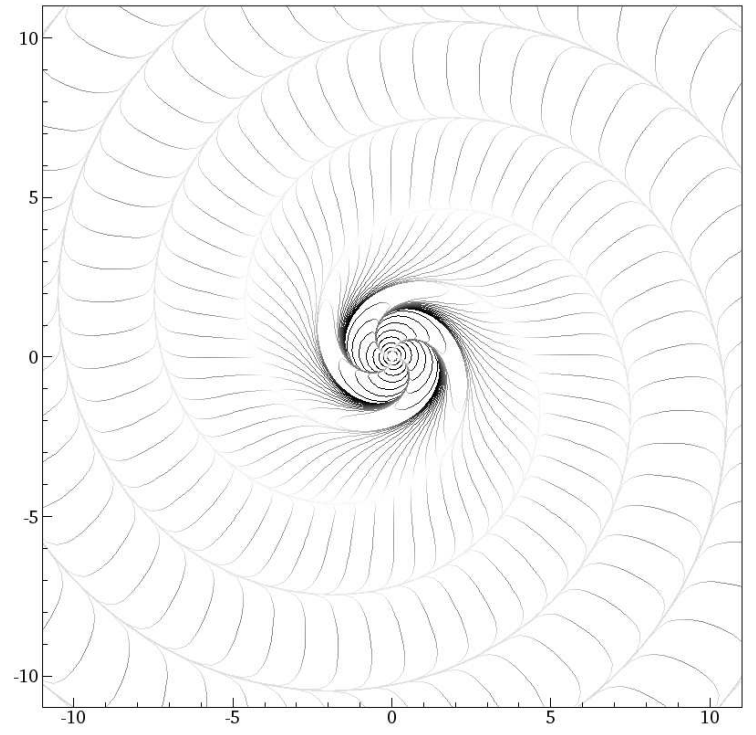

FIG. 11: Tendex lines in the equatorial plane for a rotating current quadrupole in linearized theory. The curves shown are lines of identically zero tendicity, enforced by symmetry. The lines are shaded by the absolute value of the tendicity of the other two tendex lines that cross the lines shown, but are not tangent to the plane, and have equal and opposite tendicities.

under the reflection. The diagram in Fig. 11 shows the single family of tendex lines tangent to the symmetry plane. As these curves have exactly zero tendicity, they are physically relevant only in that they denote the orientation of the other two families of tendex lines, which are not tangent to the plane, but whose projection onto the plane must be orthogonal to the curves shown (because all three curves are mutually orthogonal). The shading of the lines in Fig. 11 does not represent the tendicity of the lines drawn (which is identically zero), but rather of the other two tendex lines, which intersect the lines drawn with mutually equal and opposite tendicity. Again, this shading is rescaled by $(k r)^{5} /\left[1+(k r)^{4}\right]$. Though it isn't apparent to the eye, the strength of the tendicity grows only as $r^{4}$ near the singular point (origin), rather than $r^{5}$ as for the vorticity. As argued early in Sec. VIC, this can be interpreted intuitively as meaning that the vorticity is sourced directly from the current quadrupole, while the tendicity is sourced by induction from the time-varying vortex field.

For a rotating mass quadrupole (e.g. the quadrupole moment of an equal mass binary), the tendex lines in the plane of reflection symmetry will have precisely the same form as the rotating-current-quadrupole vortex lines of Figs. 9 and 10; see Sec. VIF. 


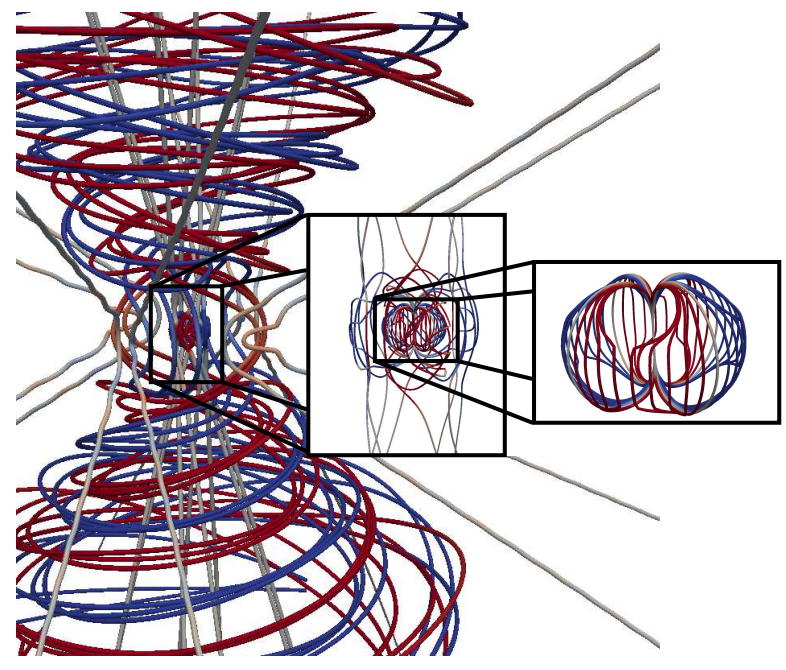

FIG. 12: For the same rotating current quadrupole as in Figs. 9 and 10: the family of vortex lines that pass orthogonally through the $x-y$ plane of reflection symmetry, color coded as in Fig. 9. In the wave zone, lines with approximately zero vorticity extend away from the source nearly radially, while lines with significant vorticity are dragged into tangled spirals by the rotation of the source. In the first inset, we see the transition between the near and wave zones. Here, lines with nearly zero vorticity escape to infinity as in the wave zone, but those with significant vorticity are drawn toward the source. The second inset delves down into the near zone, where the lines are approximately those of a stationary current quadrupole. This same figure also describes the tendex lines of a rotating mass quadrupole (see the end of Sec. VIF).

\section{Vortex lines outside the plane of reflection symmetry: transition from near zone to wave zone}

Outside the plane of reflection symmetry and in the wave zone, the extrema of the vorticity show a spiraling pattern that is the same at all polar angles. More specifically, at all polar angles $\theta$, the magnitude of the vorticity, as a function of azimuthal angle $\phi$, has four maxima; and the locations of those maxima are the same as in the equator $(\theta=\pi / 2)$. As in the equator, the maxima at fixed time $t$ spiral around at an angular rate $d \phi_{\max } / d r=-\omega$ as one moves outward in radius; and as in the equator, vortex lines collect near these spiraling maxima, and those lines too undergo spiraling behavior.

Figure 12 shows the development of this spiraling structure as one moves outward from the near zone (innermost inset) into the wave zone (outer region of figure). This figure focuses on the family of vortex lines that pass orthogonally through the $x-y$ plane of reflection symmetry. After entering the wave zone, the lines with nonnegligible vorticity (the blue and red lines) collect into a somewhat complicated spiral pattern, tangling among themselves a bit as they spiral. The gray lines with very low vorticity, by contrast, point radially outward. An animation of this rotating system can be seen at Ref. [26].

It should be noted Fig. 12, and the animation at

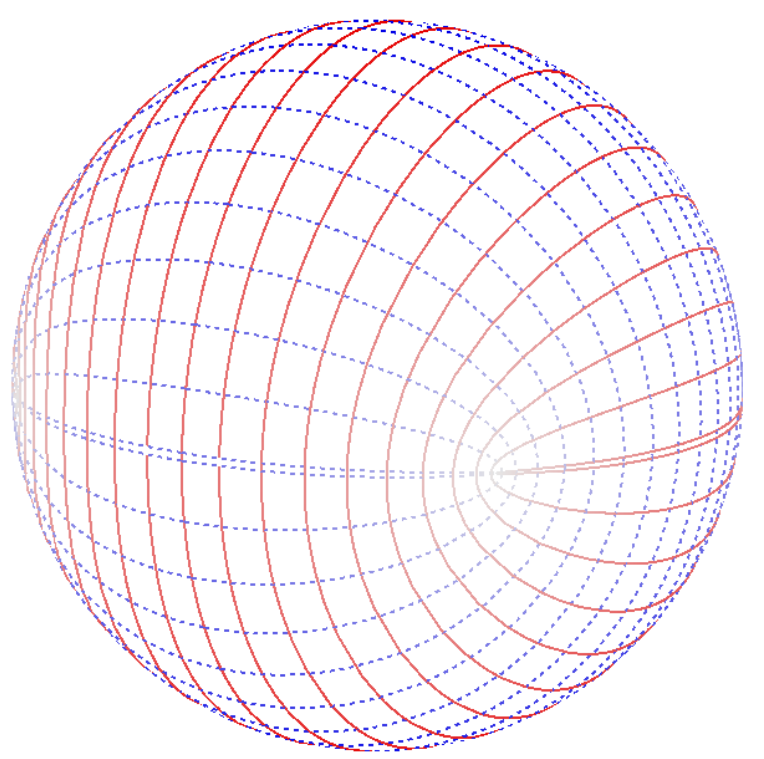

FIG. 13: (color online). Vortex lines of a time-varying current quadrupole at very large $r$. The lines are colored by the vorticity scaled by $r$, to remove the $1 / r$ falloff, but the color coding is the same as in previous figures. At very large distances from the source, the lines are transverse and live on a sphere. The third vortex line not shown is radial and has vanishing eigenvalue.

Ref. [26], represent somewhat incomplete descriptions of the structure of these field lines. The red and blue helical spirals shown in Fig. 12 do not cross one another. However, at any point in space, there must be three mutually orthogonal vortex lines, with vorticities summing to zero. Since at all points in the wave zone there is a field line of nearly zero vorticity directed in a nearly radial direction, through any point along these spirals of positive or negative vorticity, field lines of opposite vorticity must lie orthogonal to the spiral and to the approximately radial lines. As shown in the following subsection, these lines form closed loops in the far-field region.

\section{Vortex lines in the far wave zone}

In the far wave zone (strictly speaking at future null infinity), the frame-drag field becomes transverse and traceless, and takes the simple form (6.17). Of its three sets of vortex lines, one is radial (with vanishing vorticity) and the other two are tangent to a sphere of constant radius $r$ (with vorticity of equal and opposite sign). The two sets of vortex lines on the sphere have an interesting angular pattern that is shown in Fig. 13. The vortex line that lies in the equator alternates between positive and negative vorticity, going to zero at four points (one of which is shown at the front of the sphere). This line is just the limit of the spirals where vortex lines collect in Fig. 10 at very large $r$. [Further discussion of the vortex and tendex lines of radiation at large $r$ is given in 
[28], where the dual figure to Fig. 13 (tendex lines of a rotating mass quadrupole) is discussed in detail.]

How the vortex lines transition to the transverse pattern of Fig. 13 at very large $r$, from the spiraling pattern of Fig. 12 in the inner wave zone, is of considerable interest. We can explore this by examining the frame-drag field at sufficiently large radii that the $1 / r$ piece dominates over all other components, and that the $1 / r^{2}$ part of the frame-drag field may be thought of as a perturbation to the leading-order $1 / r$ part. In this region, the vortex lines show two kinds of qualitative behavior. Some of the vortex lines continue to form spirals that meander out and do not close, as in Fig. 12. There also are lines that form closed loops similar to the leading-order vortex lines of Fig. 13. We show both of these types of lines in Fig. 14. The red, solid, spiralling lines continue to collect on the maximum-vorticity spirals in the far wave zone. These lines begin to resemble the transverse lines of Fig. 13 more than the spiralling lines in the near wave zone of Fig. 12 do, because they rise and fall in polar angle as they wind around the maximum-vorticity spiral. It is only in the limit of infinite radius that these spirals close to form loops. The blue, dashed, closed lines, on the other hand, resemble the closed lines at infinity in Fig. 13 much more closely. The lines at finite $r$ do have some subtle differences between the corresponding lines at infinity: At finite radii, each individual line passes from one maximum-vorticity spiral to the other; in doing so the line must slightly increase in radius and rotate in azimuthal angle. At the large radii shown in Fig. 14, this effect is very subtle. We finally note that there are also spiralling, positive vorticity lines and closed, negative vorticity lines that we do not show to avoid visual clutter.

\section{E. Oscillating current quadrupole}

The vortex lines of an oscillating current quadrupole (this section) have a very different structure from those of the rotating current quadrupole (last section). This should not be surprising, because the two quadrupoles arise from very different physical scenarios; e.g., for the oscillating quadrupole, the ringdown following a headon collision of black holes with anti-aligned spins; and for the rotating quadrupole, the ringdown following the inspiral and merger of an extreme-kick black-hole binary. See Papers II and III of this series.

In linearized theory, one can envision an oscillating current quadrupole as produced by two particles, separated by a distance $a$ along the $x$ axis, whose spins, antialigned and pointing in the $\pm y$ direction, oscillate in magnitude as $S \cos \omega t$. The resulting quadrupole moment is [cf. Eq. $(5.14)]$

$$
\mathcal{S}_{x y}=\mathcal{S}_{y x}=S a \cos \omega t .
$$

The frame-drag and tidal fields, and thence vortex and

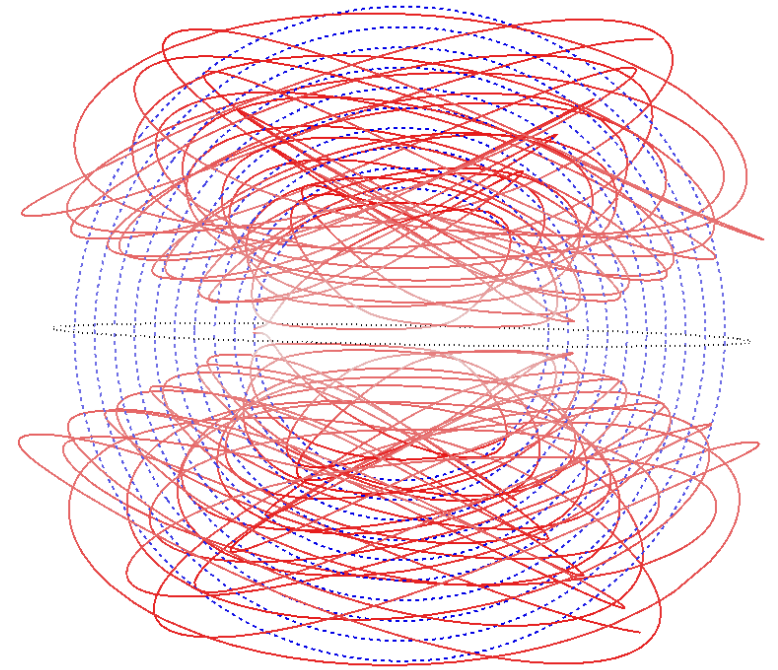

FIG. 14: (color online). Vortex lines of a rotating current quadrupole at sufficiently large $r$ that the $1 / r^{2}$ part of the frame-drag field may be thought of as a perturbation to the transverse vortex lines of Fig. 13. The lines are colored by the vorticity as in that figure. We also show a black dotted circle in the equatorial plane to identify this plane. The red lines shown here continue to collect on the maximum-vorticity spiral, but they oscillate much more in polar angle than do the similar lines shown in the near wave zone in Fig. 12. The blue lines shown here form closed loops that pass from one positive vorticity spiral to the next. This family of lines more closely resembles the transverse lines of Fig. 13, though in the limit of infinite radius, the spiralling lines will also close to form transverse lines on the sphere. There are also spiralling positive vorticity (blue) lines and closed-loop, negative vorticity (red) lines, but to keep the figure from appearing muddled, we do not show them.

tendex lines, for this current quadrupole can be computed from Eqs. (6.15) and (6.16).

As for the rotating quadrupole, the $x-y$ plane of reflection symmetry contains two families of vortex lines, and a third family passes orthogonally through that plane. The in-plane vortex lines are depicted in Figs. 15 and 16 using the same color conventions as for the rotating quadrupole (Figs. 9 and 10). Figure 15 shows the region of the near zone that is difficult to see in Fig. 16, an equivalent figure that spans a larger region of the $x-y$ plane. As one can see from the figures, the two families of vortex lines, solid red (negative vorticity) and dashed blue (positive vorticity) have the same pattern, but are rotated by $\pi / 2=90^{\circ}$.

The way in which the gravitational waves are generated differs greatly from the rotating current quadrupole of the previous section. In the near zone, the two sets of vortex lines form a static quadrupole pattern (identical to the near-zone rotating quadrupole of Fig. 9, but rotated by $\pi / 4$ due to the orientation of the spins). In the transition zone, the vortex lines form distorted loops that head away from the origin, along the lines $y= \pm x$, in al- 


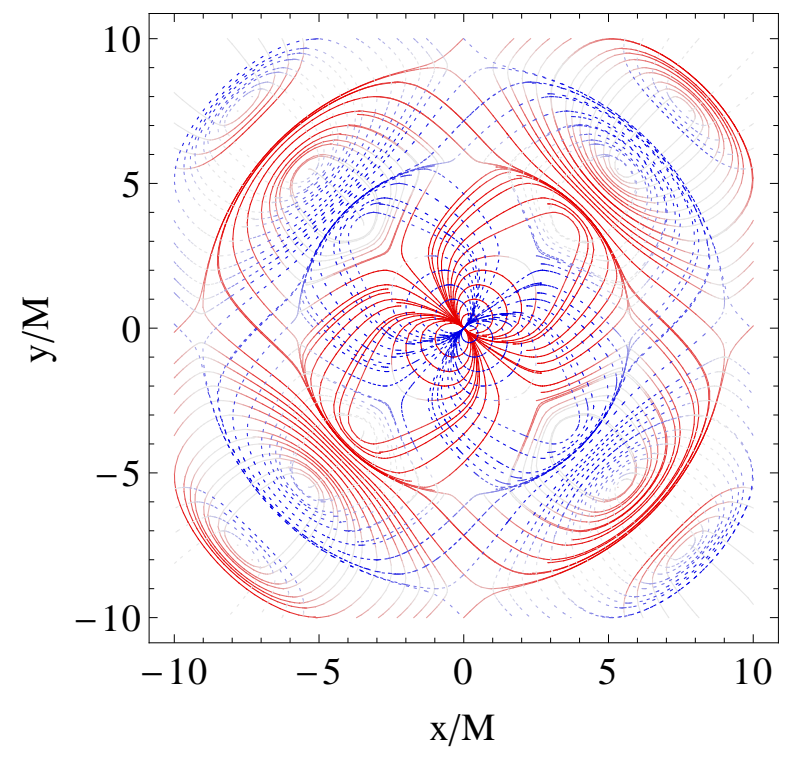

FIG. 15: (color online). For an oscillating current quadrupole in linearized theory: two families of vortex lines in the plane of reflection symmetry (the $x-y$ plane). The color coding is the same as for the rotating current quadrupole, Fig. 9. The vortex lines begin, near the origin, like the static quadrupole pattern of Fig. 6. The effects of time retardation cause the pattern to stretch making larger rectangular loops in the transition zone. As time passes and the quadrupole oscillates, these loops detach from the origin and propagate out into the wave zone. This same figure also describes the tendex lines of an oscillating mass quadrupole (see the end of Sec. VIF).

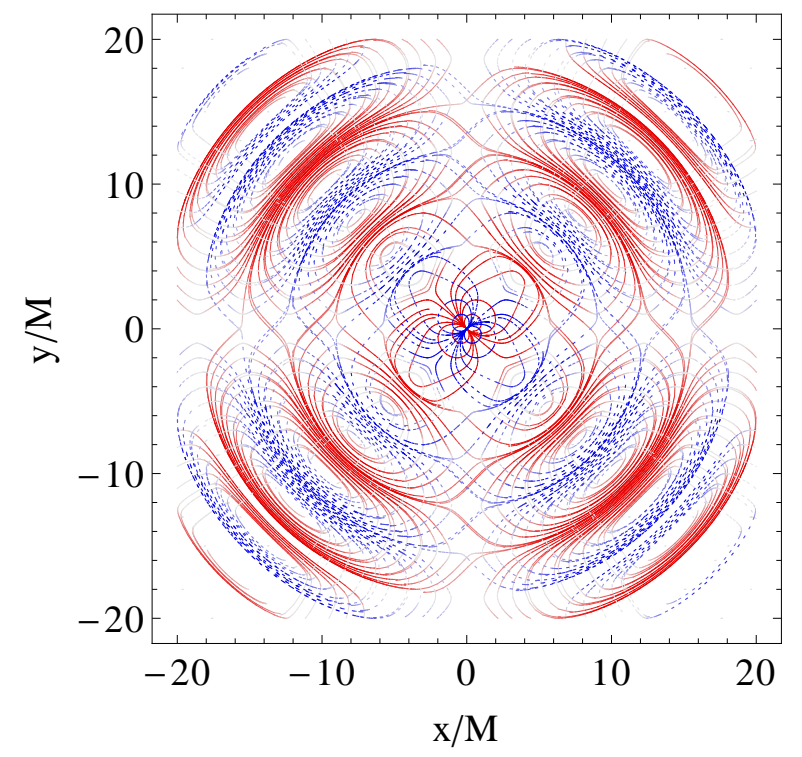

FIG. 16: (color online). Same as Fig. 15, but zoomed out to show the wave zone. Further from the source, the loops take on a more regular alternating pattern of gravitational waves. The coloring shows that the vorticity is strongest at the fronts and backs of the loops, where the vortex lines are transverse to the direction of propagation. In the regions of the closed loops that extend radially, the field is weak (as one would expect for a transverse gravitational wave). ternating fashion. As they extend into the wave zone, the lines form two qualitatively different kinds of loops. The majority of the loops reside only in one of the four quadrants of the equatorial plane, but there are also loops that pass through all four quadrants, staying near the regions of maximum vorticity, where lines collect at the gravitational wave crests. For both types of loops, they maintain the same wavelength, but the wave front becomes wider at larger radii, as they become gravitational waves. The portion of a loop transverse to the radial direction (the direction of propagation) has strong vorticity, as one would expect for a gravitational wave; in the radial portion of the loop, the vorticity is weak. Each cycle of the oscillating quadrupole casts off another set of vortex loops as the near zone region passes through zero vorticity, and the loops travel outward towards infinity. This illustrates clearly the manner in which the near zone vortex pattern generates gravitational waves in the far zone through its dynamics.

As with the rotating current quadrupole, one can envision the equatorial vortex line of Fig. 13 as the limit of the wavefronts of the planar vortex lines in Fig. 16 at large distances. It is again of interest to understand how the vortex lines outside the equatorial plane become the remaining vortex lines in Fig. 13. To do so, we will make reference to Fig. 17, which shows the vortex lines at a distance sufficiently large that the $1 / r^{2}$ portions of the frame-drag field can be thought of as a small perturbation to the transverse vortex lines of Fig. 13. We show only the three-dimensional analog of the lines that pass through all four quadrants in the equatorial plane, and do not show the lines that remain in just one octant (analogous to the loops that remain in one quadrant in the equatorial plane) to keep the figure as simple as possible.

Near the poles, these vortex lines have nearly the same structure as the purely transverse lines of Fig. 13; it is only near the equator that the lines begin to differ. As the lines approach the equator, they also increase in radius, due to the $1 / r^{2}$ parts of the frame-drag field. In doing so, they pass from one gravitational wave crest to the next, and the lines sharply turn during their passage between successive crests. The portion of the line on this next crest runs nearly parallel to the equator, until it begins moving slightly inward (again due to the $1 / r^{2}$ parts of the frame-drag field). As it then sharply turns again, it returns to the original crest and begins heading back toward the poles. This sharp turning happens on both sides of the sphere, which causes the lines to form the closed loops that reside in either the northern or the southern hemisphere in Fig. 17. Only in the limit that $r$ goes to infinity do the radial perturbations vanish, and the loops in the northern and southern hemisphere connect to form the transverse pattern in Fig. 13. 


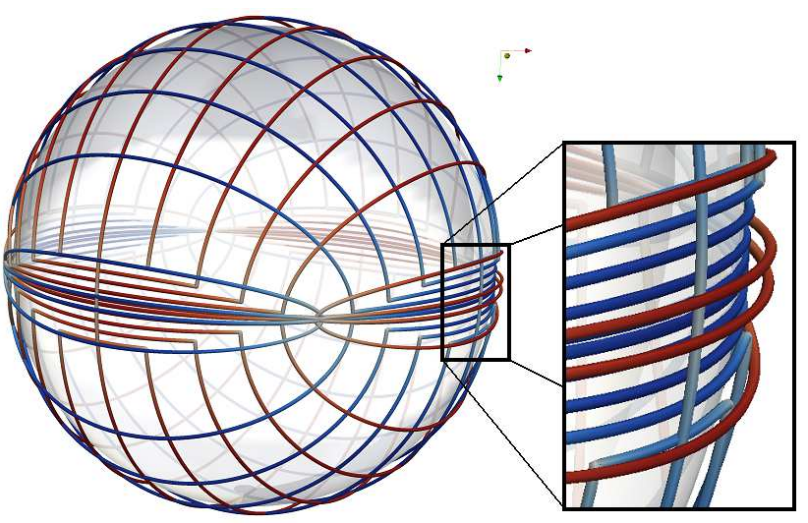

FIG. 17: (color online). Vortex lines of an oscillating current quadrupole at sufficiently large $r$ that the $1 / r^{2}$ part of the frame-drag field may be thought of as a perturbation to the transverse vortex lines of Fig. 13. The lines are colored in the same way as that figure, and the pattern of the lines around the poles is nearly identical to the transverse lines of Fig. 13. Near the equator, the $1 / r^{2}$ perturbation causes the lines to bend and form closed loops that reside in either the northern or the southern hemisphere. The blue horizontal lines in the blow up inset should be compared with dense blue (dashed) bundles in Fig. 16, and red lines with the red bundles immediately outside of the blue ones.

\section{F. Wave generation by a time varying mass quadrupole}

A time varying mass quadrupole moment $\mathcal{I}_{p q}(t)$ gives rise to metric perturbations of flat space given by the terms proportional to $\mathcal{I}_{p q}(t-r) / r$ and its derivatives in Eqs. (8.13) of [36]. It is straightforward to calculate that the frame-drag field for these metric perturbations is

$$
\mathcal{B}_{i j}=\epsilon_{p q(i}\left[\left(\frac{{ }^{(1)} \mathcal{I}_{p k}}{r}\right)_{, j) k q}-\left(\frac{{ }^{(3)} \mathcal{I}_{j) p}}{r}\right)_{, q}\right] .
$$

Notice that this mass-quadrupolar frame-drag field is the same as the current-quadrupolar tidal field (6.16), with the current quadrupole moment $\mathcal{S}_{p q}$ replaced by $-\frac{3}{4} \mathcal{I}_{p q}$; cf. the duality relations (2.14) and (2.18). Correspondingly, the vortex lines of this mass quadrupole will be the same as the tendex lines of the equivalent current quadrupole.

The mass quadrupole's tidal field can be deduced from its frame-drag field (6.21) by using the third of the Maxwell-like equations (2.15). The result is

$$
\begin{aligned}
\mathcal{E}_{i j}= & \frac{1}{2}\left[-\left(\frac{\mathcal{I}_{p q}}{r}\right)_{, p q i j}+\epsilon_{i p q}\left(\frac{{ }^{(2)} \mathcal{I}_{p m}}{r}\right)_{, q n} \epsilon_{j m n}\right. \\
& \left.+2\left(\frac{{ }^{(2)} \mathcal{I}_{p(i}}{r}\right)_{, j) p}-\left(\frac{{ }^{(4)} \mathcal{I}_{i j}}{r}\right)\right] .
\end{aligned}
$$

Alternatively, this mass quadrupolar tidal field can be deduced from the current quadrupolar frame-drag field
(6.15) by using the duality relation $\mathcal{S}_{p q} \rightarrow+\frac{3}{4} \mathcal{I}_{p q}$ [Eqs. (2.14) and (2.18)].

As a result, the tendex lines of this mass quadrupole will be the same as the vortex lines of the current quadrupole, Figs. 9 - 10 and 12 - 16, with the red (solid) lines describing tidal stretching, and the blue (dashed) lines, tidal squeezing.

\section{G. Slow-Motion Binary system made of identical, nonspinning point particles}

As a final example of a weakly gravitating system, we investigate the tendex lines of a Newtonian, equal mass binary made of nonspinning point particles in a circular orbit. We assume a separation $a$ between particles that is large compared to their mass $M$, so the orbital velocity $v=\frac{1}{2} \sqrt{M / a}$ is small compared to the speed of light ("slow-motion binary").

Close to the binary, where retardation effects are negligible, the tidal field is given by the Newtonian expression $\mathcal{E}_{j k}=\Phi_{, j k}[$ Eq. (5.4)], with $\Phi$ the binary's Newtonian gravitational potential

$$
\Phi=-\frac{M_{A}}{\left|\boldsymbol{x}-\boldsymbol{x}_{A}\right|}-\frac{M_{B}}{\left|\boldsymbol{x}-\boldsymbol{x}_{B}\right|} .
$$

Here $M_{A}=M_{B}=M / 2$ are the particles' masses with $M$ the total mass, and $\boldsymbol{x}_{A}$ and $\boldsymbol{x}_{B}$ are the locations of particles, which we take to be on the $x$ axis, separated by a distance $a$.

In Fig. 18, we show the near-zone tendex lines associated with this potential's tidal field, color coded in the usual way (see the figure's caption). Close to each particle, the tendex lines resemble those of a static, spherically symmetric object. Moving further from the particle, one can see the effects of the particle's companion, bending and compressing the lines. At radii $r \gtrsim a$, the Newtonian potential and tidal field can be expanded in multipole moments with the monopole and quadrupole dominating. At $r>>a$, the monopole dominates and the tendex lines become those of a single spherical body.

The binary's orbital angular velocity is $\omega=\sqrt{M / a^{3}}$ (Kepler's formula), and the binary emits gravitational waves with angular frequency $2 \omega$, reduced wavelength $\lambda=1 /(2 \omega)=\frac{1}{2} \sqrt{a^{3} / M}$, and wavelength $\lambda=2 \pi \lambda$. As a concrete example, we choose the particles' separation to be $a=20 M$; then $\lambda=\sqrt{5} a \simeq 2.24 a$, and $\lambda=2 \pi \sqrt{5} a \simeq$ $14 a$.

Figure 19 shows tendex lines in this binary's orbital plane, focusing on the transition and wave zones $r \gtrsim \lambda=$ $2.24 a$ (outside the solid black circle). The shapes and colors of the tendex lines in this figure can be understood in terms of the binary's multipole moments:

In the transition zone and wave zone, $r \gtrsim \lambda$, the tidal field is the sum of a nonradiative monopolar piece with magnitude $\mathcal{E}_{M} \simeq M / r^{3}$, and a quadrupolar piece with magnitude $(1 / r) \partial^{4} \mathcal{I} / \partial t^{4} \simeq(2 \omega)^{4}\left(\frac{1}{4} M a^{2}\right) / r \simeq 4 M^{3} / a^{4} r$; higher order moments are negligible. The two moments 


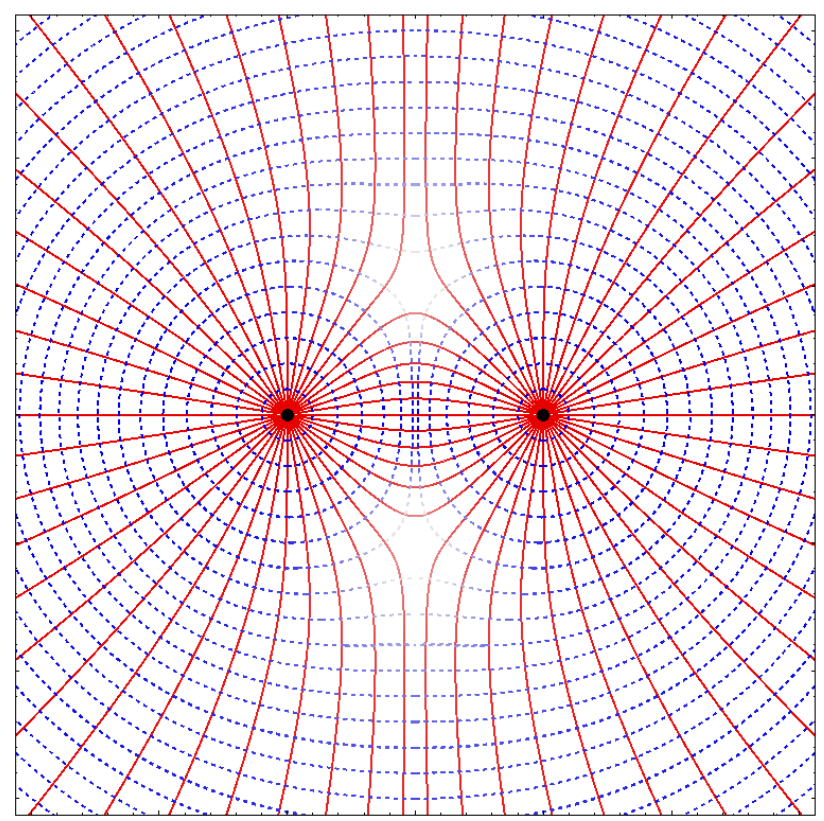

FIG. 18: (color online). For a weak-gravity binary made of identical nonspinning point particles, in the near zone where retardation is negligible: two families of tendex lines lying in a plane that passes through the two particles (e.g. the orbital plane). The red (solid) curves are lines with negative tendicity, and the blue (dashed) curves have positive tendicity. The color intensity of the curves represents the magnitude of the tendicity, rescaled by $r_{A}^{3} r_{B}^{3} /\left[M^{3}\left(r_{A}^{3}+r_{B}^{3}\right)\right]$, where $r_{A}$ and $r_{B}$ are the distances to the particles, to remove the tendicity's radial die out. Near each particle, the tendex lines resemble those of an isolated spherical body; as one moves closer to the particle's companion, the lines bend in response to its presence. At radii large compared to the particles' separation $a$, the binary's monopole moment comes to dominate, and the tendex lines resemble those of a single isolated spherical body.

contribute about equally at radius $r=\frac{1}{2} a^{2} / M=10 a$ (dotted black circle in the figure). The (nonradiative) monopole moment, with its red radial and blue circular tendex lines, dominates inside this circle. The (radiative) quadrupole moment dominates outside the circle, so there the tendicity is significant (strong red and blue) only when the tendex lines are transverse; and strong red alternates, radially, with strong blue as the waves propagate radially. Ultimately, at very large radii (far outside the domain of Fig. 19), the quadrupole moment will totally dominate, and the tendex-line pattern will become that of a rotating quadrupole, depicted in Fig. 10.

Figure 20 shows the tendex lines for this same binary, with the same parameters, in three dimensions, i.e. above and below the equatorial plane. In the inner region, the monopole moment dominates so the red (stretching) tendex lines are nearly radial, and the blue (squeezing) tendex lines are nearly circular, centered on the binary. As one moves outward, the radiative quadrupole moment begins to distort these radial and circular tendex lines, and then at large radii, the now-dominant quadrupole

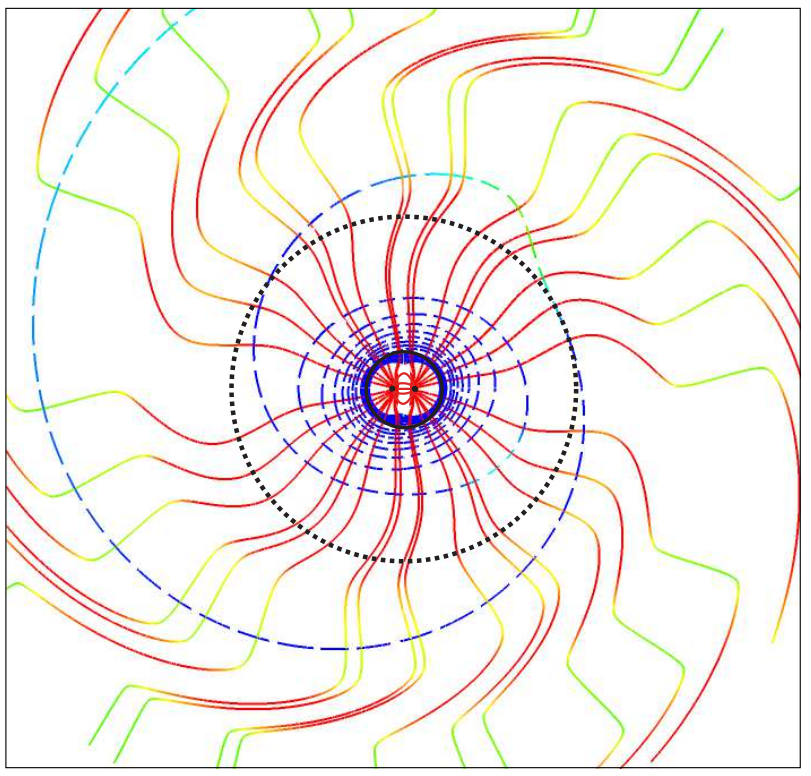

FIG. 19: (color online). Tendex lines in the orbital plane of the same binary as Fig. 18, with separation $a=20 M$ (where $M$ is the total mass), focusing on the transition and wave zones $r \gtrsim \lambda=2.24 a$. The solid black circle has radius $\lambda$. The colors are fixed by the tendicity weighted by $\omega r$ so as to scale out the $1 / r$ falloff in the wave zone (with dark blue strongly positive, dark red strongly negative, and light green near zero). Inside the dotted black curve ( $\left.r=\frac{1}{2} a^{2} / M=10 a\right)$, the binary's (nonradiative) monopole moment dominates, $\mathcal{E} \simeq M / r^{2}$, and the red (stretching) tendex lines are nearly radial. Outside the dotted black curve, the (radiative) quadrupole moment dominates, $\mathcal{E} \simeq 4 M^{3} / a^{4} r$, and the tendex lines are strong (significant tendicity) only where they are approximately transverse to the radial direction.

moment drives them into the same spiraling pattern as we have seen in Fig. 12 for the tendex lines of a rotating, pure mass quadrupole.

\section{CONCLUSIONS}

In this paper, we have focused on the "electric" and "magnetic" parts of the Weyl curvature tensor, $\mathcal{E}_{i j}$ and $\mathcal{B}_{i j}$, and have given them the names tidal field and framedrag field, based on their roles in producing tidal gravitational accelerations and differential frame dragging. Being parts of the Riemann tensor, these fields are well defined (though slicing dependent) in strong-gravity situations such as the near zone of colliding black holes. For this reason, and because they embody the full vacuum Riemann tensor and are easily visualized, $\mathcal{E}_{i j}$ and $\mathcal{B}_{i j}$ are powerful tools for exploring the nonlinear dynamics of spacetime curvature (geometrodynamics).

As tools for visualizing $\mathcal{E}_{i j}$ and $\mathcal{B}_{i j}$, we have introduced tendex and vortex lines (the integral curves of the eigenvectors of $\mathcal{E}_{i j}$ and $\mathcal{B}_{i j}$ ), along with their tendicities and 


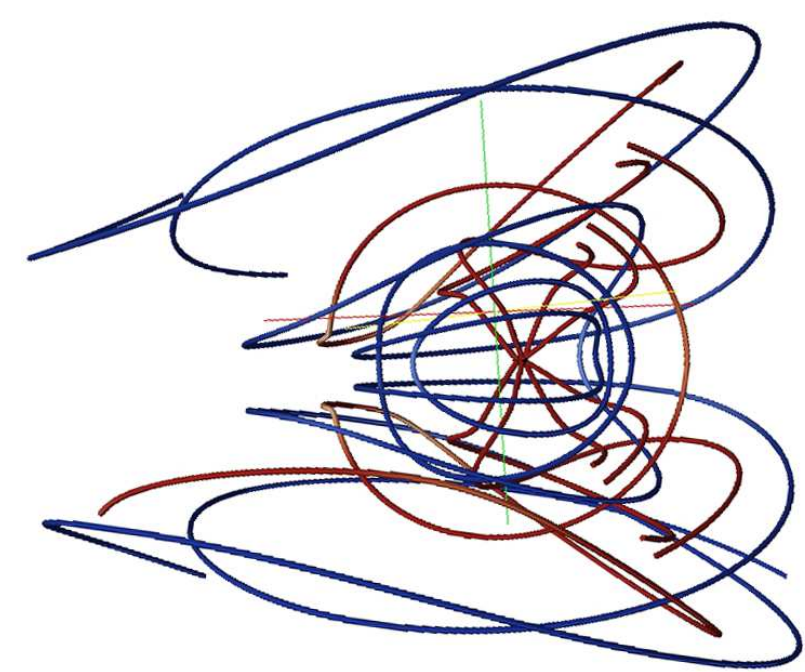

FIG. 20: (color online). Tendex lines outside the (central, horizontal) orbital plane, for the same binary and parameters as Fig. 19. In the inner region, the binary's monopole moment dominates, $\mathcal{E} \simeq M / r^{2}$, so the red (stretching) tendex lines are nearly radial and the blue (squeezing) tendex lines are nearly circular. At larger radii, the (radiative) quadrupole moment begins to be significant and then dominate, so the tendex lines begin to spiral outward as for the rotating quadrupole of Fig. 12 .

vorticities (the eigenvectors' eigenvalues). The tendex and vortex lines are gravitational analogs of electric and magnetic field lines. Moreover, just as the electromagnetic field tensor is fully determined by its electric and magnetic field lines, together with their field-line densities (which encode the lengths of the electric and magnetic vectors), so the vacuum Riemann curvature tensor is fully determined by its tendex and vortex lines, together with their colors (which encode the tendicities and vorticities as in Fig. 19).

In terms of their transformation properties, the $\left(\mathcal{E}_{i j}\right.$, $\mathcal{B}_{i j}$ ) pair is strictly analogous to the pair of electric and magnetic 3-vector fields: they are components of a 4tensor, divided into two groups in a slicing dependent manner. We are confident that this mild and transparent form of frame dependence will not prevent our tendex and vortex concepts from becoming useful tools for studying geometrodynamics, any more than the frame dependence of electric and magnetic fields and field lines have been impeded from being useful tools for studying electromagnetism in flat or curved spacetime.

Using various examples from linearized gravity, for which analytical formulas are available, we have plotted color-coded tendex and vortex lines, and thereby we have gained insight into the behaviors of the tidal and framedrag fields. This intuition from weak-gravity examples will be of great value when studying strongly gravitating systems in asymptotically flat spacetimes, e.g. binary black holes. This is because, in the weak-gravity region of spacetime outside such strong-gravity systems, linearized gravity is a good approximation. More specifically:

For stationary, strongly gravitating systems (e.g. stationary black holes and neutron stars), the tendex and vortex lines in their asymptotic, weak-gravity regions will be well approximated by our linearized-theory results in Sec. V (and, perhaps in some cases, extensions to higher multipoles).

For oscillatory, strongly gravitating systems (e.g. binary black holes and oscillating neutron stars), the wave zones' tendex and vortex lines will be well approximated by those of our examples in Sec. VI, and their extensions. Whether the system has strong gravity or weak gravity, its wave-zone field lines are controlled by radiative multipole moments that are tied to the system's near-zone dynamics.

As one moves inward through the weak-gravity wave zone into the near zone and the region of strong gravity, the details of the field lines and the system's dynamics may be quite different for strong-gravity systems than for our weak-gravity examples. Nevertheless it seems likely that in all cases, the gravitational waves will be generated by dynamical motions of near-zone tendexes and vortexes (regions of strong tendicity and vorticity). By exploring that near-zone tendex/vortex dynamics, we can gain deep physical insight into nonlinear spacetime curvature. This will be a central theme of Papers II and III in this series.

Whatever may be a source's strong-field dynamics, it will be useful to focus on the imprints that the strong-field dynamics leaves on the tendex/vortex structures in the strong-to-weak-gravity transition region. Those transition-region tendex/vortex imprints will govern spacetime curvature throughout the asymptotic, weak-gravity region, and in particular will govern the radiative multipole moments that control the emitted gravitational waves. Moreover, the imprinted structures in the strong-to-weak-gravity transition region may turn out to have some sort of effective dynamics that can be captured by simple analytical models and can become a powerful tool for generating approximate gravitational waveforms, e.g. for use in gravitational-wave data analysis.

\section{Acknowledgments}

We thank John Belcher, Larry Kidder, Richard Price, and Saul Teukolsky for helpful discussions. Our simulations have been performed using the Spectral Einstein Code (SpEC) [45]. This research was supported by NSF grants PHY-0601459, PHY-0653653, PHY0960291, PHY-0969111, PHY-1005426, PHY-1068881 and CAREER grant PHY-0956189, by NASA grants NNX09AF97G and NNX09AF96G, and by the Sherman Fairchild Foundation, the Brinson Foundation, and the David and Barbara Groce fund. 


\section{Appendix A: The Newman Penrose Formalism}

In this appendix we give the connection between the electric and magnetic parts of the Weyl tensor $\mathcal{E}$ and $\mathcal{B}$, and the five Newman-Penrose (NP) curvature scalars [46].

The NP formalism [46] is especially useful for expressing the gravitational wave content of a dynamical spacetime at asymptotic null infinity. It is also a crucial foundation for the study of black hole perturbations and for the Petrov classification of vacuum spacetimes, both of which will naturally make contact with the study of vortexes and tendexes. In order to make contact with numerical simulations, we will need to understand the connection between the NP formalism and gravitational waves propagating on a flat background, as discussed in Sec. VI A.

Because we use the opposite metric signature to that of the original Newman-Penrose paper [46] and the widely used Penrose-Rindler book [32], our sign conventions for the NP quantities and for Eqs. (A3) and (A8) below differ from theirs. Ours are the same as in [33].

To begin with, we define an orthonormal tetrad $\vec{e}_{\hat{\alpha}}=$ $\left(\vec{e}_{\hat{0}}, \vec{e}_{\hat{1}}, \vec{e}_{\hat{2}}, \vec{e}_{\hat{3}}\right)$ with time basis vector $\vec{e}_{\hat{0}}=\vec{u}$ orthogonal to our chosen foliation's spacelike hypersurfaces, and with the spatial basis vectors $\vec{e}_{\hat{1}}, \vec{e}_{\hat{2}}, \vec{e}_{\hat{3}}$ lying in those hypersurfaces. We use this tetrad to build a complex null tetrad for use in the NP formalism:

$$
\begin{array}{cc}
\vec{l}=\frac{1}{\sqrt{2}}\left(\vec{e}_{\hat{0}}+\vec{e}_{\hat{1}}\right), & \vec{n}=\frac{1}{\sqrt{2}}\left(\vec{e}_{\hat{0}}-\vec{e}_{\hat{1}}\right), \\
\vec{m}=\frac{1}{\sqrt{2}}\left(\vec{e}_{\hat{2}}+i \vec{e}_{\hat{3}}\right), & \vec{m}^{*}=\frac{1}{\sqrt{2}}\left(\vec{e}_{\hat{2}}-i \vec{e}_{\hat{3}}\right) .
\end{array}
$$

By projecting the Weyl tensor onto this null basis, we construct the complex Weyl scalars,

$$
\begin{aligned}
& \Psi_{0}=C_{\mu \nu \rho \sigma} l^{\mu} m^{\nu} l^{\rho} m^{\sigma}, \\
& \Psi_{1}=C_{\mu \nu \rho \sigma} l^{\mu} n^{\nu} l^{\rho} m^{\sigma}, \\
& \Psi_{2}=C_{\mu \nu \rho \sigma} l^{\mu} m^{\nu} m^{* \rho} n^{\sigma}, \\
& \Psi_{3}=C_{\mu \nu \rho \sigma} l^{\mu} n^{\nu} m^{* \rho} n^{\sigma}, \\
& \Psi_{4}=C_{\mu \nu \rho \sigma} n^{\mu} m^{* \nu} n^{\rho} m^{* \sigma} .
\end{aligned}
$$

Using the null tetrad (A1) built from our orthonormal tetrad, we can express the spatial orthonormal components of the electric and magnetic parts of the Weyl tensor in terms of the Weyl scalars as follows:

$$
\begin{aligned}
& \mathcal{E}_{\hat{\hat{a} \hat{b}}}+i \mathcal{B}_{\hat{a} \hat{b}} \\
= & {\left[\begin{array}{ccc}
2 \Psi_{2} & -\left(\Psi_{1}-\Psi_{3}\right) & i\left(\Psi_{1}+\Psi_{3}\right) \\
* & \frac{\Psi_{0}+\Psi_{4}}{2}-\Psi_{2} & -\frac{i}{2}\left(\Psi_{0}-\Psi_{4}\right) \\
* & * & -\frac{\Psi_{0}+\Psi_{4}}{2}-\Psi_{2}
\end{array}\right] }
\end{aligned}
$$

(cf. Eq (3.65) of [33], where the differences are due to differing conventions on both $\mathcal{B}$ and our null tetrad). In
Eq. (A3), the rows and columns are ordered as $\hat{1}, \hat{2}, \hat{3}$ and the entries indicated by $*$ are given by the symmetry of the matrix.

The entries in Eq. (A3) can be derived in a straightforward manner from the definitions of $\mathcal{E}$ and $\mathcal{B}$, Eqs. (2.2a) and $(2.2 \mathrm{~b})$, and the definitions of the Weyl scalars, Eqs. (A2a)-(A2e). For example, we have

$$
\begin{aligned}
\mathcal{E}_{\hat{1} \hat{1}} & =R_{\hat{1} \hat{0} \hat{1} \hat{0}}=\frac{1}{2}\left(R_{\hat{r} l \hat{r} l}+2 R_{\hat{r} l \hat{r} n}+R_{\hat{r} n \hat{r} n}\right) \\
& =\frac{1}{4}\left(R_{n l n l}-2 R_{n l l n}+R_{l n l n}\right)=R_{l n l n}
\end{aligned}
$$

where we have used the symmetry properties of the Riemann tensor to eliminate and combine many terms. This result is not obviously equal to any of the Weyl scalars, but note that

$$
\begin{aligned}
R_{l n l n} & =-R_{n l n}^{n}=R_{n n l}^{n}=-\left(R_{n l l}^{l}+R_{n m l}^{m}+R^{m^{*}{ }_{n m^{*} l}}\right) \\
& =-R_{m^{*} n m l}-R_{m n m^{*} l}=R_{l m m^{*} n}+R_{l m^{*} m n} \\
& =\Psi_{2}+\Psi_{2}^{*},
\end{aligned}
$$

where we have used the fact that in the null tetrad basis $\left\{\vec{l}, \vec{n}, \vec{m}, \vec{m}^{*}\right\}$, indices are raised and lowered with the metric components

$$
g_{\alpha \beta}=g^{\alpha \beta}=\left(\begin{array}{cccc}
0 & -1 & 0 & 0 \\
-1 & 0 & 0 & 0 \\
0 & 0 & 0 & 1 \\
0 & 0 & 1 & 0
\end{array}\right)
$$

and the fact that the Ricci tensor vanishes in vacuum spacetimes. Similar manipulations give

$$
\begin{aligned}
B_{\hat{1} \hat{1}} & =\frac{1}{2} \epsilon_{\hat{1}}^{\hat{p} \hat{q}} R_{\hat{p} \hat{q} \hat{1} \hat{0}}=R_{\hat{2} \hat{3} \hat{1} \hat{0}}=-i R_{m^{*} m l n} \\
& =i\left(R_{l m n m^{*}}+R_{l m^{*} m n}\right)=i\left(-\Psi_{2}+\Psi_{2}^{*}\right)
\end{aligned}
$$

so we see that $\mathcal{E}_{\hat{1} \hat{1}}+i \mathcal{B}_{\hat{1} \hat{1}}=2 \Psi_{2}$. Similar computations give all of the entries of Eq. (A3).

We will often have reason to consider the "horizon tendicity" and "horizon vorticity." These are the values of $\mathcal{E}$ and $\mathcal{B}$ projected normal to the 2-dimensional event horizon of a spacetime containing a black hole, evaluated at the horizon. If the inward normal to the horizon is denoted $\boldsymbol{N}$ and we choose the vector $\boldsymbol{e}_{\hat{1}}$ such that it coincides with $-\boldsymbol{N}$ at the horizon, then we immediately have the useful result

$$
\begin{aligned}
\Psi_{2} & =\frac{1}{2}\left(\mathcal{E}_{N N}+i \mathcal{B}_{N N}\right) \\
& =\frac{1}{2}\left(\mathcal{E}_{i j}+i \mathcal{B}_{i j}\right) N^{i} N^{j}
\end{aligned}
$$

which we will use in our studies of analytic and numerical spacetimes containing horizons (papers II and III in this series). 
[1] R. Owen, J. Brink, Y. Chen, J. D. Kaplan, G. Lovelace, K. D. Matthews, D. A. Nichols, M. A. Scheel, F. Zhang, A. Zimmerman, et al., Phys. Rev. Lett. 106, 151101 (2011).

[2] J. A. Wheeler, Geometrodynamics (Academic Press, New York, 1963).

[3] J.A. Wheeler, in Relativity, Groups and Topology (Les Houches Summer School, 1963), edited by B. DeWitt and C. DeWitt (Gordon and Breach, New York, 1964), pp. 325-520.

[4] C. W. Misner, Phys. Rev. 118, 1110 (1960).

[5] D. R. Brill and R. W. Lindquist, Phys. Rev. 131, 471 (1963).

[6] S. Hahn and R. Lindquist, Ann. Phys. (NY) 29, 304 (1964).

[7] J. Weber, Phys. Rev. 117, 306 (1960).

[8] J. M. Centrella, J. G. Baker, B. J. Kelly, and J. R. van Meter, Rev. Mod. Phys. 82, 3069 (2010).

[9] B. C. Barish and R. Weiss, Phys. Today 52, 44 (1999).

[10] D. Sigg and the LIGO Scientific Collaboration, Class. Quantum Grav. 25, 114041 (2008).

[11] F. Acernese et al. (Virgo Collaboration), Class. Quantum Grav. 25, 184001 (2008).

[12] K. Kuroda and the LCGT Collaboration, Class. Quantum Grav. 27, 084004 (2010).

[13] G. M. Harry (for the LIGO Scientific Collaboration), Class. Quantum Grav. 27, 084006 (2010).

[14] C. W. Misner, K. S. Thorne, and J. A. Wheeler, Gravitation (Freeman, New York, New York, 1973).

[15] L. Smarr, Phys. Rev. D 7, 289 (1973).

[16] D. Marolf, Gen. Rel. Grav. 31, 919 (1999).

[17] U. Jacob and T. Piran, Class. Quantum Grav. 23, 4035 (2006).

[18] J. D. Romano and R. H. Price, Class. Quantum Grav. 12, 875 (1995).

[19] M. Bondarescu, M. Alcubierre, and E. Seidel, Class. Quantum Grav. 19, 375 (2002).

[20] H. Chan, Class. Quantum Grav. 23, 225 (2006).

[21] L. Rezzolla, R. P. Macedo, and J. L. Jaramillo, Phys. Rev. Lett. 104, 221101 (2010), arxiv:1003.0873.

[22] J. L. Jaramillo, R. P. Macedo, P. Moesta, and L. Rezzolla (2011), arxiv:1108.0060.

[23] J. L. Jaramillo, R. P. Macedo, P. Moesta, and L. Rezzolla
(2011), arxiv:1108.0061.

[24] J. L. Jaramillo (2011), arxiv:1108.2408.

[25] J. W. Belcher and S. Olbert, Am. J. Phys. 71, 220 (2003).

[26] http://www.black-holes.org/curQuadVortex.html.

[27] http://www.black-holes .org/antiAliPoints.html.

[28] A. Zimmerman, D. A. Nichols, and F. Zhang, Phys. Rev. D 84, 044037 (2011).

[29] F. B. Estabrook and H. D. Wahlquist, Journal of Mathematical Physics 5, 1629 (1964).

[30] C. Schmid, Phys. Rev. D 79, 064007 (2009).

[31] R. Maartens and B. A. Bassett, Class. Quantum Grav. 15, 705 (1998).

[32] R. Penrose and W. Rindler, Spinors and Space-time, Volume 1 (Cambridge University Press, Cambridge, 1992).

[33] H. Stephani, D. Kramer, M. MacCallum, C. Hoenselaers, and E. Herlt, Exact solutions of Einstein's field equations (2003).

[34] H. Friedrich, Class. Quantum Grav. 13, 1451 (1996).

[35] A. Anderson, Y. Choquet-Bruhat, and J. W. York, Jr., Topol. Meth. Nonlin. Anal. 10, 353 (1997).

[36] K. S. Thorne, Rev. Mod. Phys. 52, 299 (1980).

[37] R. D. Blandford and K. S. Thorne, Applications of Classical Physics (California Institute of Technology, 2008), URL http://www.pma.caltech.edu/Courses/ ph136/yr2008/.

[38] J. D. Kaplan, D. A. Nichols, and K. S. Thorne, Phys. Rev. D 80, 124014 (2009).

[39] R. L. Forward, Proc. IRE 49, 892 (1961).

[40] D. Keppel, D. A. Nichols, Y. Chen, and K. S. Thorne, Phys. Rev. D 80, 124015 (2009), arXiv:0902.4077.

[41] G. Lovelace, Y. Chen, M. Cohen, J. D. Kaplan, D. Keppel, K. D. Matthews, D. A. Nichols, M. A. Scheel, and U. Sperhake, Phys. Rev. D 82, 064031 (2010).

[42] K. S. Thorne, R. H. Price, and D. A. MacDonald, Black Holes: The Membrane Paradigm (Yale University Press, New Haven and London, 1986).

[43] D. R. Fiske, J. G. Baker, J. R. van Meter, D.-I. Choi, and J. M. Centrella, Phys. Rev. D 71, 104036 (2005).

[44] R. Penrose, Ann. Hum. Genet., Lond. 42, 435 (1979).

[45] http://www.black-holes.org/SpEC.html.

[46] E. Newman and R. Penrose, Journal of Mathematical Physics 3, 566 (1962). 